This is the submitted version of the article:

Lodi Rizzini, A.; Krull, C.; Mugarza, A.; Balashov, T.; Nistor, C.; Piquerel, R.; Klyatskaya, S.; Ruben, M.; Sheverdyaeva, P.M.; Moras, P.; Carbone, C.; Stamm, C.; Miedema, P.S.; Thakur, P.K.; Sessi, V.; Soares, M.; Yakhou-Harris, F.; Cezar, J.C.; Stepano. Coupling of single, double, and triple-decker metal-phthalocyanine complexes to ferromagnetic and antiferromagnetic substrates. Surface Science, (2014). 630. null: $361-.10 .1016 /$ j.susc.2014.07.008.

Available at: https://dx.doi.org/10.1016/j.susc.2014.07.008 


\title{
Coupling of single, double, and triple-decker metal-phthalocyanine complexes to ferromagnetic and antiferromagnetic substrates
}

\author{
Alberto Lodi Rizzini $^{\mathrm{a}}$, Cornelius Krulla ${ }^{\mathrm{a}}$, Aitor Mugarza ${ }^{\mathrm{a}}$, Timofey Balashov ${ }^{\mathrm{a}}$, Corneliu Nistor $^{\mathrm{a}, \mathrm{b}}$, Raoul Piquerel $^{\mathrm{a}}$, \\ Svetlana Klyatskaya ${ }^{\mathrm{c}}$, Mario Ruben ${ }^{\mathrm{c}, \mathrm{d}}$, Polina M. Sheverdyaeva ${ }^{\mathrm{e}}$, Paolo Moras ${ }^{\mathrm{e}}$, Carlo Carbone ${ }^{\mathrm{e}}$, Christian Stamm $^{\mathrm{b}}$, \\ Piter S. Miedema ${ }^{\mathrm{f}}$, Pardeep K. Thakur ${ }^{\mathrm{g}}$, Violetta Sessi ${ }^{\mathrm{g}}$, Marcio Soares ${ }^{\mathrm{g}}$, Flora Yakhou-Harris ${ }^{\mathrm{g}}$, Julio C. Cezar ${ }^{\mathrm{g}}$, \\ Sebastian Stepanow ${ }^{\mathrm{b}}$, Pietro Gambardella $\mathrm{a}^{\mathrm{a}, \mathrm{b}, \mathrm{h},}$ \\ ${ }^{a}$ Catalan Institute of Nanoscience and Nanotechnology (ICN2), UAB Campus, E-08193 Barcelona, Spain \\ ${ }^{b}$ Department of Materials, ETH Zürich, Hönggerbergring 64, CH-8093 Zürich, Switzerland \\ ${ }^{c}$ Institute of Nanotechnology, Karlsruhe Institute of Technology (KIT), D-76344 Eggenstein-Leopoldshafen, Germany \\ ${ }^{d}$ Institute de Physique et Chimie de Matériaux de Strasbourg (IPCMS), UMR 7504, CNRS-Université de Strasbourg, F-67034 Strasbourg, France \\ ${ }^{e}$ Istituto di Struttura della Materia, Consiglio Nazionale delle Ricerche, 34012 Trieste, Italy \\ ${ }^{f}$ Debye Institute of Nanomaterials Science, Utrecht University, 3584 CA Utrecht, The Netherlands \\ ${ }^{g}$ European Synchrotron Radiation Facility, BP 220, F-38043 Grenoble, France \\ ${ }^{h}$ Institució Catalana de Recerca i Estudis Avançats (ICREA), E-08010 Barcelona, Spain
}

\begin{abstract}
We report a survey of the magnetic properties of metal-organic complexes coupled to ferromagnetic and antiferromagnetic surfaces. Using element-resolved x-ray magnetic circular dichroism, we investigate the magnetism of single, double, and triple-decker phthalocyanines focusing on $\mathrm{MnPc}, \mathrm{TbPc}_{2}$, and $\mathrm{Tb}_{2} \mathrm{Pc}_{3}$ deposited on $\mathrm{Ni}, \mathrm{Mn}$, and $\mathrm{CoO}$ thin films. Depending on the number of Pc ligands, we find that the metal ions within the molecules couple either parallel or antiparallel to a ferromagnetic substrate. Whereas single-decker complexes such as MnPc form a unique magnetic entity with ferromagnetic films, the intrinsic single molecule magnet properties of $\mathrm{TbPc}_{2}$ and $\mathrm{Tb}_{2} \mathrm{Pc}_{3}$ remain largely unaltered. $\mathrm{TbPc}_{2}$ deposited on perpendicularly magnetized Ni films exhibits enhanced magnetic stability compared to $\mathrm{TbPc}_{2}$ in molecular crystals, opposite to $\mathrm{TbPc}_{2}$ deposited on in-plane magnetized Ni. Depending on the competition between uniaxial anisotropy, superexchange, and Zeeman interaction, the magnetic moment of $\mathrm{TbPc}_{2}$ can be aligned parallel or antiparallel to that of the substrate by modulating the intensity of an external magnetic field. This occurs also for $\mathrm{Tb}_{2} \mathrm{Pc}_{3}$, but the substrate-induced exchange coupling in triple-decker molecules is found to be short-ranged, that is, limited to the $\mathrm{Tb}$ ion closer to the ferromagnetic surface. Finally, we discuss the conditions required to establish exchange bias between molecules and antiferromagnetic substrates. We show that $\mathrm{TbPc}_{2}$ deposited on antiferromagnetic Mn thin films exhibits both exchange bias and enhanced coercivity when field cooled parallel to the out-of-plane easy axis. However, exchange bias does not extend to all molecules on the surface. On oxide antiferromagnets such as $\mathrm{CoO}$ we find no evidence of exchange bias for either $\mathrm{TbPc}_{2}$ or MnPc.
\end{abstract}

Key words: phthalocyanines, exchange bias, hybrid metal-organic interfaces, XMCD

\section{Introduction}

A fascinating topic in surface science is the fabrication and study of materials that have no counterpart in bulk systems. Magnetic multilayers, in particular, provide textbook examples of unusual properties that arise from the combination of diverse elements as well as from size and interface effects [1]. In the last three decades, the investigation of magnetic coupling in such systems [2,3] has led to significant advances in the ability to control their magnetization and electrical properties, which is paramount for information recording technology and spintronic applications [4].

Following recent interest in molecular spintronics [5, 6], several phenomena well-known for metal- and oxide-based multilayers, such as the giant magnetoresistance [7, 8], tunneling

Email addresses: pietro.gambardella@mat.ethz.ch (Pietro Gambardella) magnetoresistance [9], exchange spring magnetism [10], and exchange bias [11], have become of interest also for molecular systems [12, 13, 14, 15, 16]. Molecular magnets offer exciting prospects in this field due to their small size, well-defined structure, and flexibility of chemical synthesis. Yet their use in practical devices is hindered by magnetic relaxation effects as well as by the difficulty of interfacing and embedding the molecules in solid-state electronic platforms. These difficulties have motivated recent efforts to couple magnetic molecules to ferromagnetic (FM) and antiferromagnetic (AFM) metal layers.

Because of the need to preserve a clean interface between the molecules and substrate, experiments in this area have followed a classical surface science approach, starting from the preparation of magnetic films on single crystal susbtrates, to the sublimation of molecular layers in ultra high vacuum (UHV) and the characterization by surface-sensitive techniques such 
Table 1: Metal-organic complexes on magnetic substrates investigated by different techniques. The type of coupling is indicated as FM (ferromagnetic), AFM (antiferromagnetic), and NC (not coupled).

\begin{tabular}{|c|c|c|c|c|}
\hline Molecule & Substrate & Technique & Coupling & Ref. \\
\hline \multicolumn{5}{|c|}{ Ferromagnetic substrates } \\
\hline $\mathrm{MnPc}, \mathrm{FePc}, \mathrm{CuPc}$ & $\mathrm{Fe}(100)$ & SPMDS $^{a}$ & FM & [17] \\
\hline $\mathrm{MnPc}$ & $\mathrm{Co} / \mathrm{Cu}(100)$ & $\mathrm{XMCD}^{b}$ & FM & [18] \\
\hline $\mathrm{MnPc}, \mathrm{FeF}_{16} \mathrm{Pc}$ & $\mathrm{O} / \mathrm{Co} / \mathrm{Cu}(100)$ & XMCD & AFM & [19] \\
\hline $\mathrm{MnPc}$ & $\mathrm{Ni} / \mathrm{Ag}(100)$ & XMCD & $\mathrm{FM}$ & This work \\
\hline $\mathrm{FePc}, \mathrm{CoPc}, \mathrm{CuPc}$ & $\mathrm{Co} / \mathrm{Cu}(100)$ & SPUPS $^{c}$ & FM & [20] \\
\hline $\mathrm{FePc}$ & $\mathrm{Co} / \mathrm{Cu}(100)$ & XMCD & $\mathrm{FM}$ & [21] \\
\hline $\mathrm{FePc}$ & $\mathrm{O} / \mathrm{Co} / \mathrm{Cu}(100)$ & XMCD & AFM & [22] \\
\hline $\mathrm{CoPc}$ & $\mathrm{Co} / \mathrm{Cu}(111)$ & SPSTM $^{d}$ & $\mathrm{FM}$ & [23] \\
\hline $\mathrm{CoPc}$ & $\mathrm{Fe} / \mathrm{W}(110)$ & SPSTM & & [24] \\
\hline $\mathrm{CoPc}$ & $\mathrm{Fe} / \mathrm{Cu}(111)$ & XMCD & FM & [21] \\
\hline MnTPP & $\mathrm{Co} / \mathrm{Au}(111)$ & XMCD & FM & [25] \\
\hline MnTPP & $\mathrm{O} / \mathrm{Co} / \mathrm{Cu}(100)$ & XMCD & AFM & [26] \\
\hline CoTPP & $\mathrm{Ni} / \mathrm{Cu}(100)$ & XMCD & FM & [27] \\
\hline FeTPP & $\mathrm{Ni} / \mathrm{Cu}(100)$ & XMCD & FM & [28] \\
\hline FeOEP & $\mathrm{Ni}, \mathrm{Co} / \mathrm{Cu}(100)$ & XMCD & FM & [29] \\
\hline FeOEP & $\mathrm{O} / \mathrm{Ni}, \mathrm{O} / \mathrm{Co} / \mathrm{Cu}(100)$ & XMCD & AFM & [30] \\
\hline CoOEP & graphene/Ni/W(110) & XMCD & AFM & [31] \\
\hline $\mathrm{Cu}$-tetraazaporphyrin & $\mathrm{Fe}_{3} \mathrm{O}_{4} / \mathrm{MgO}(100)$ & XMCD & AFM & [32] \\
\hline $\mathrm{TbPc}_{2}$ & $\mathrm{Ni} / \mathrm{Cu}(100), \mathrm{Ni} / \mathrm{Ag}(100)$ & XMCD & AFM & This work and [15] \\
\hline $\mathrm{TbPc}_{2}$ & $\mathrm{O}, \mathrm{Li} \mathrm{Ni} / \mathrm{Cu}(100)$ & XMCD & AFM & This work and [15] \\
\hline $\mathrm{TbPc}_{2}$ & $\mathrm{Co} / \operatorname{Ir}(111)$ & SPSTM & & [33] \\
\hline $\mathrm{TbPc}_{2}$ & $\mathrm{Co} / \mathrm{Cu}(100)$ & XMCD & AFM & [34] \\
\hline $\mathrm{TbPc}_{2}$ & $\mathrm{La}_{0.3} \mathrm{Sr}_{0.7} \mathrm{Mn}_{O 3}$ & XMCD & $\mathrm{NC}$ & [35] \\
\hline $\mathrm{Tb}_{2} \mathrm{Pc}_{3}$ & $\mathrm{Ni} / \mathrm{Cu}(100)$ & XMCD & AFM & This work \\
\hline \multicolumn{5}{|c|}{ Antiferromagnetic substrates } \\
\hline $\mathrm{MnPc}$ & $\mathrm{CoO} / \mathrm{Ag}(100)$ & XMCD & $\mathrm{NC}$ & This work \\
\hline $\mathrm{TbPc}_{2}$ & $\mathrm{CoO} / \mathrm{Ag}(100)$ & XMCD & $\mathrm{NC}$ & This work and [16] \\
\hline $\mathrm{TbPc}_{2}$ & $\mathrm{Mn} / \operatorname{Ag}(100)$ & XMCD & $\mathrm{FM}$ & This work and [16] \\
\hline
\end{tabular}

${ }^{\bar{a}}$ Spin-polarized metastable deexcitation spectroscopy

${ }^{b} \mathrm{X}$-ray magnetic circular dichroism

${ }^{c}$ Spin-polarized ultraviolet photoelectron spectroscopy

${ }^{d}$ Spin-polarized scanning tunneling microscopy

(a)

(b)

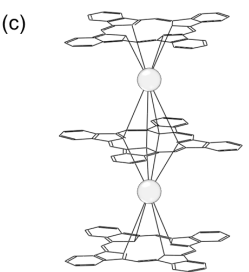

Figure 1: Structure of (a) single, (b) double, and (c) triple-decker metalphthalocyanines.

as X-ray magnetic circular dichroism (XMCD) and scanning tunneling microscopy (STM). Investigations have been carried out mainly on planar metal-organic complexes such as metalphthalocyanines (MPc), metal-tetraphenylporphyrins (MTPP), and octaethylporphyrins (MOEP), as shown in Table I. These

aromatic molecules contain a macrocycle tetrapyrrole structure with a central transition-metal ion coordinated to the $\mathrm{N}$ atoms of the pyrroles, and differ in the type of end groups attached to the pyrroles. Owing to their robust structure as well as versatile chemistry, such molecules have assumed the role of model systems to study the interaction of metal-organic complexes with metal surfaces [36]. Their flat adsorption geometry facilitates self-assembly into ordered layered structures [37, 38, 39, 40] and the bonding of both the central metal ion and organic ligands to the substrate. This gives rise to charge transfer from the substrate to the $\pi$-orbitals of the macrocycle and $d$-orbitals of the metal ion, which enhances [41] or suppresses [42] the magnetic moment of the molecules depending on the symmetry of the $d$-states and the sign of the $d-\pi$ exchange coupling [36]. Despite such differences, for all planar molecules investigated to date, the interaction between the magnetic moment of the 


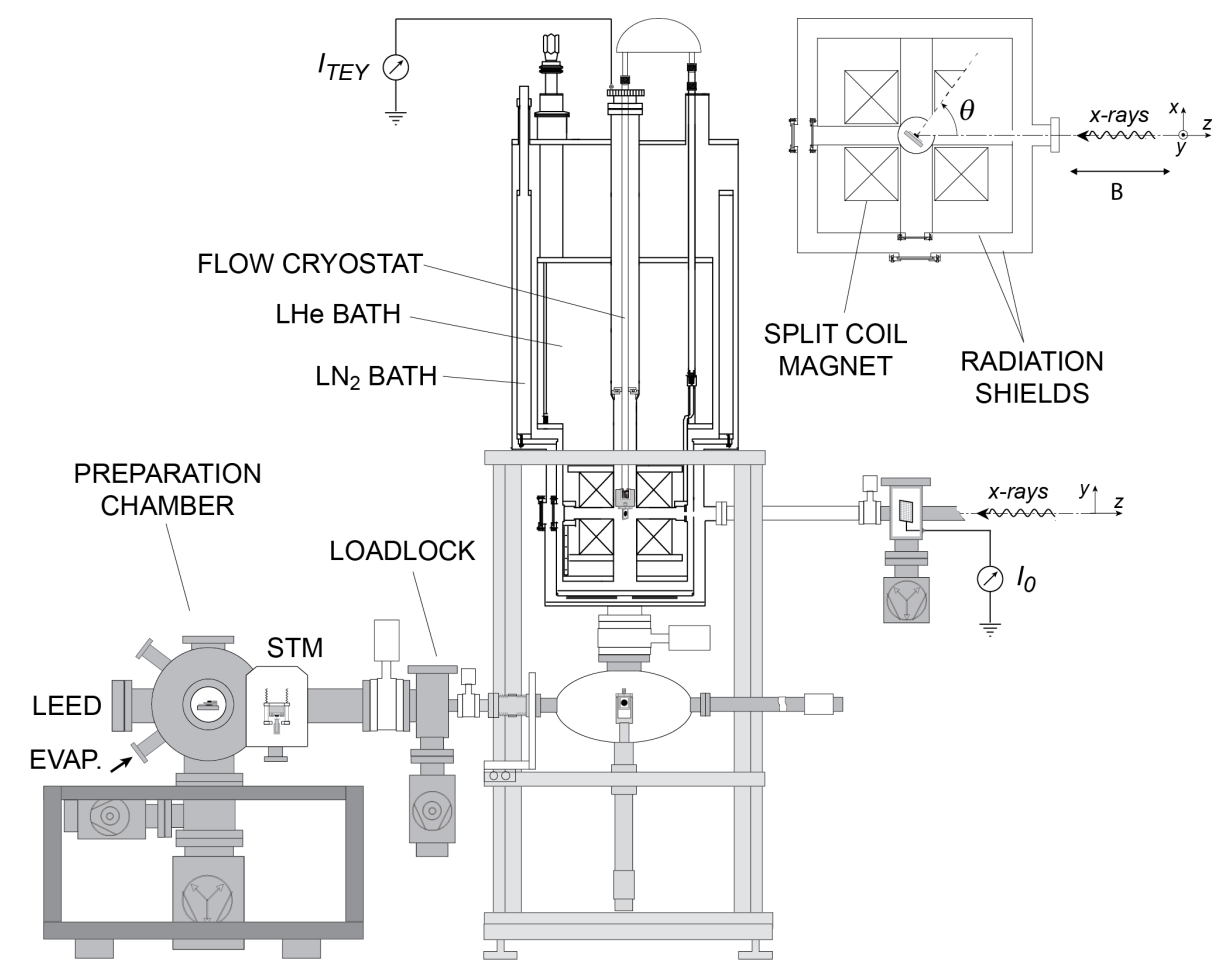

Figure 2: Schematics of the experimental setup and sample geometry for x-ray linear and circular dichroism measurements on beamline ID08 (now ID32) of the ESRF.

molecules and that of metal substrates has been found to be FM (see Table I). This has been attributed to the direct exchange path between the central metal ion and the substrate atoms as well as to an indirect superexchange path via the $\mathrm{N}$ atoms [29, 43]. Only when $\mathrm{O}$ or graphene is intercalated between the molecules and the substrates the coupling has been found to be AFM (see Table I).

Fewer experiments have been dedicated to nonplanar molecules, notably to $\mathrm{TbPc}_{2}[15,16,33,34,35]$, a single molecule magnet (SMM) consisting of a $\mathrm{Tb}$ ion sandwiched between two Pc ligands [44, 45], as shown in Fig. 1 (b). Compared to other SMM, this molecule has the advantage that it can be evaporated in UHV while preserving its structure and SMM properties [46, 47]. When deposited onto a FM surface, the Tb magnetic moment couples antiparallel to the substrate magnetization. However, because the $\mathrm{Tb}$ ion does not bond directly to the surface, the strength of the exchange coupling is such that its magnetic moment can be aligned either parallel or antiparallel to the substrate by varying the strength of an external magnetic field [15]. This makes $\mathrm{TbPc}_{2}$ a very interesting system for the realization of molecular spin valves since its magnetic properties remain different from the substrate. Further, the possibility to form multilayered compounds where metal ions are stacked between sandwich-type Pc oligomers, as in $\mathrm{Tb}_{2} \mathrm{Pc}_{3}$ and related multiple-decker complexes [48, 49], makes these systems of interest to study the effects of magnetic coupling in large polynuclear molecules that extend away from the magnetic interface.

This paper focuses on the exchange coupling properties of planar and nonplanar metal-organic molecules to FM and
AFM substrates. We present XMCD measurements of single$(\mathrm{MnPc})$, double- $\left(\mathrm{TbPc}_{2}\right)$, and triple-decker $\left(\mathrm{Tb}_{2} \mathrm{Pc}_{3}\right)$ phthalocyanines as representative examples of stacked $\pi$-conjugate molecules in which the distance between the magnetic ions and a FM or AFM surface progressively increases (Fig. 1). We consider Ni as FM substrate for all molecules and $\mathrm{CoO}$ and $\mathrm{Mn}$ as AFM substrates. The remaining of this paper is organized as follows: Section 2 describes the experimental setup and XMCD measurements. Section 3 reports the characterization of molecular adsorption on different substrates by STM and x-ray linear dichroism. The magnetic behavior of $\mathrm{MnPc}, \mathrm{TbPc}_{2}$, and $\mathrm{Tb}_{2} \mathrm{Pc}_{3}$ on Ni films is reported in Sect. 4, 5, and 6, respectively. Finally, Sect. 7 describes molecular exchange bias on AFM surfaces and Sect. 8 summarizes the results.

\section{Experimental}

The experiments reported in this work are based on XMCD measurements of the molecule and substrate magnetization. Because of its element-resolving power [1] and sensitivity to low concentrations of magnetic atoms [50, 51], XMCD is ideally suited to study molecular systems on surfaces. The measurements were performed at beamline ID08 of the European Synchrotron Radiation Facility (ESRF). A scheme of the experimental setup, which includes a liquid He cryostat with high field magnet for XMCD measurements and a dedicated preparation chamber is shown in Fig. 2. The samples were prepared in-situ by molecular beam evaporation of $\mathrm{MnPc}, \mathrm{TbPc}_{2}$, 

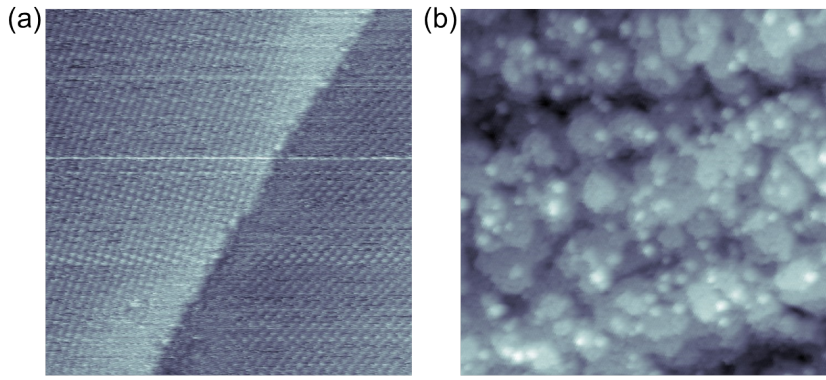

(c)
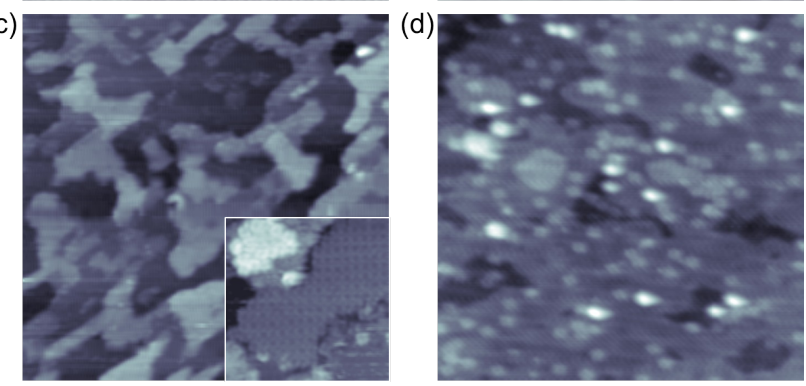

Figure 3: STM images of (a) 1 ML MnPc on $\mathrm{Ag}(100)$. Image size: $75 \times 75 \mathrm{~nm}^{2}$. (b) $0.05 \mathrm{ML} \mathrm{TbPc}_{2}$ on $13 \mathrm{ML} \mathrm{Ni} / \mathrm{Cu}(100)$. Image size: $65 \times 65 \mathrm{~nm}^{2}$. (c) $3 \mathrm{ML} \mathrm{CoO} / \mathrm{Ag}(100)$. Image size: $150 \times 150 \mathrm{~nm}^{2}$. Inset: $\mathrm{TbPc}_{2}$ on $3 \mathrm{ML}$ $\mathrm{CoO} / \mathrm{Ag}(100), 25 \times 25 \mathrm{~nm}^{2}$. (d) $\mathrm{TbPc}_{2}$ on $3 \mathrm{ML} \mathrm{Mn} / \mathrm{Ag}(100)$. Image size: $50 \times 50 \mathrm{~nm}^{2}$. All images have been recorded at room temperature using the setup shown in Fig. 2.

and $\mathrm{Tb}_{2} \mathrm{Pc}_{3}$ on magnetic thin films deposited onto singlecrystal $\mathrm{Ag}(100)$ and $\mathrm{Cu}(100)$ substrates. The MnPc molecules were purchased from Sigma-Aldrich with $99.9 \%$ purity. The terbium bis-(phthalocyanine) and homoleptic, homodinuclear tris(phthalocyaninato) complexes were synthesised according to modified templating reactions of the phthalonitrile precursor with terbium acetylacetonate, as described in Refs. [52] and [53].

The whole sample preparation procedure was carried out in UHV with a base pressure of $2 \times 10^{-10}$ mbar. The molecules were degassed in UHV for at least 24 hours prior to evaporation and the metal substrates cleaned by repeated Ar sputtering cycles and annealed to $500{ }^{\circ} \mathrm{C}$. FM films with inplane magnetic anisotropy were obtained by depositing $\mathrm{Ni}$ on $\operatorname{Ag}(100)$ [15], whereas films with out-of-plane anisotropy were obtained by depositing Ni layers thicker than 10 ML (monolayer) on $\mathrm{Cu}(100)[54,55]$. AFM CoO layers of thickness ranging from 3 to $15 \mathrm{ML}$ were grown by evaporating Co in a pure oxygen atmosphere of $10^{-7}$ mbar on $\operatorname{Ag}(100)$ [56], whereas AFM Mn layers were obtained by direct deposition on $\mathrm{Ag}(100)$ at room temperature [16]. STM was used to calibrate the coverage of the magnetic thin films and molecules as well as to provide indications on the morphology of the molecule/metal layers. After preparation, the samples were transferred to the cryostat chamber for $\mathrm{x}$-ray measurements without breaking vacuum.

The X-ray absorption measurements were carried out by measuring the total electron yield (TEY) of the sample during scans of the photon energy over the $L_{2,3}$ absorption edges of Mn, Co, and Ni, the $M_{4,5}$ absorption edges of $\mathrm{Tb}$, and the $K$ absorption
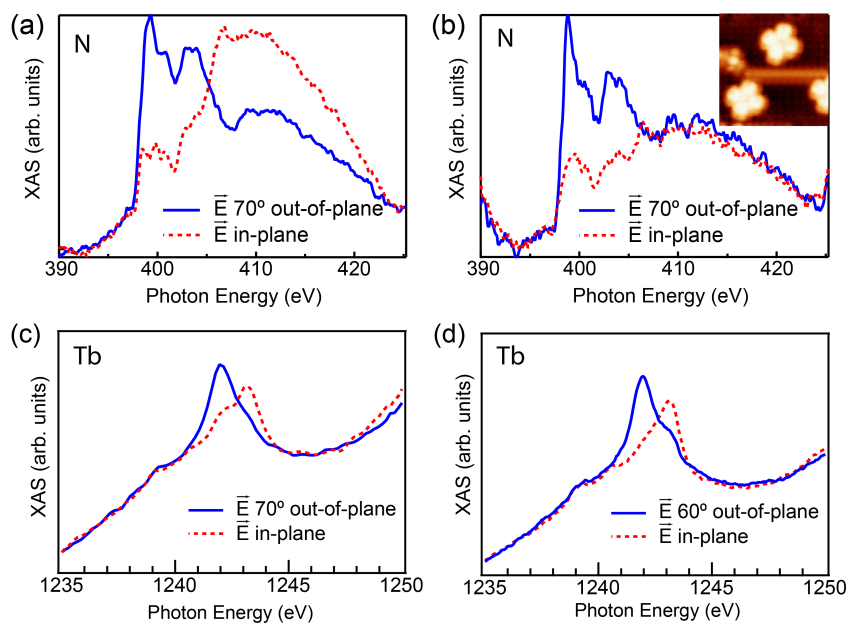

Figure 4: Linearly polarized x-ray absorption spectra of (a) $\mathrm{TbPc}_{2} / \mathrm{Ni} / \mathrm{Cu}(100)$ and (b) $\mathrm{TbPc}_{2}$ deposited on $c(2 \times 2) \mathrm{O} / \mathrm{Ni} / \mathrm{Cu}(100)$ recorded at the Nitrogen $K$-edge with $\mathbf{E}$ parallel to the surface plane (red dashed line) and $70^{\circ}$ out-ofplane (blue solid line). The x-ray incidence angle is $\theta=70^{\circ}$ in both cases. Inset: $\mathrm{STM}$ image of $\mathrm{TbPc}_{2} / c(2 \times 2) \mathrm{O} / \mathrm{Ni} / \mathrm{Cu}(100)$, image size $5 \times 5 \mathrm{~nm}^{2}$. (c) Linearly polarized $\mathrm{x}$-ray absorption spectra of $\mathrm{TbPc}_{2} / \mathrm{Ni} / \mathrm{Cu}(100)$ and (d) $\mathrm{Tb}_{2} \mathrm{Pc}_{3} / \mathrm{Ni} / \mathrm{Cu}(100)$ recorded at the $\mathrm{Tb} M_{5}$ edge.

edge of N. The photoelectron current of the sample was normalized by the incoming photon flux measured by the TEY of a thin Au mesh placed upstream from the cryostat ( $I_{0}$ in Fig. 2). A magnetic field $\mathbf{B}$ of up to $\pm 5 \mathrm{~T}$ was applied parallel to the $\mathrm{X}$-ray incidence direction at an angle $\theta$ with respect to the sample normal and used for the XMCD measurements as well as for field cooling of the samples. XMCD spectra were obtained by subtracting consecutive $\mathrm{X}$-ray absorption spectra (XAS) recorded for parallel $\left(I^{+}\right)$and antiparallel $\left(I^{-}\right)$alignment of the photon helicity and sample magnetization.

Element-resolved magnetization curves were measured by XMCD in two different ways. The first method consisted in performing two (forward and backward) sweeps of the applied magnetic field, one for positive and the other for negative circularly polarized light. During the first sweep, only the pre-edge and the maximum intensity $I^{+}$at either the $L_{3}$ or $M_{5}$ edge were measured, thus optimizing the acquisition time for the energy point where the XMCD is maximum. The peak intensity values were divided by the pre-edge intensity at each field in order to eliminate the dependence of the TEY on the sample orientation and magnetic field. Then a second sweep was performed to measure $I^{-}$. Finally, the normalized intensity from the first loop was subtracted from the second in order to obtain the XMCD loop. This is a well-established method to measure magnetization curves using XMCD [57, 58, 59], which gives better results than just plotting the peak $I^{ \pm}$intensity as a function of field as done for relatively thick metal films [60]. The second method consisted in measuring complete XMCD spectra at each value of the magnetic field, averaging up to $16 \mathrm{XMCD}$ spectra per point, and plotting the maximum XMCD intensity at either the $L_{3}$ or $M_{5}$ edge normalized by the average intensity $\left(I^{+}+I^{-}\right) / 2$ at each point. This method is much more time consuming compared to the first, but gives a self-consistent magnetization measurement at each point without the need of subtracting consecu- 

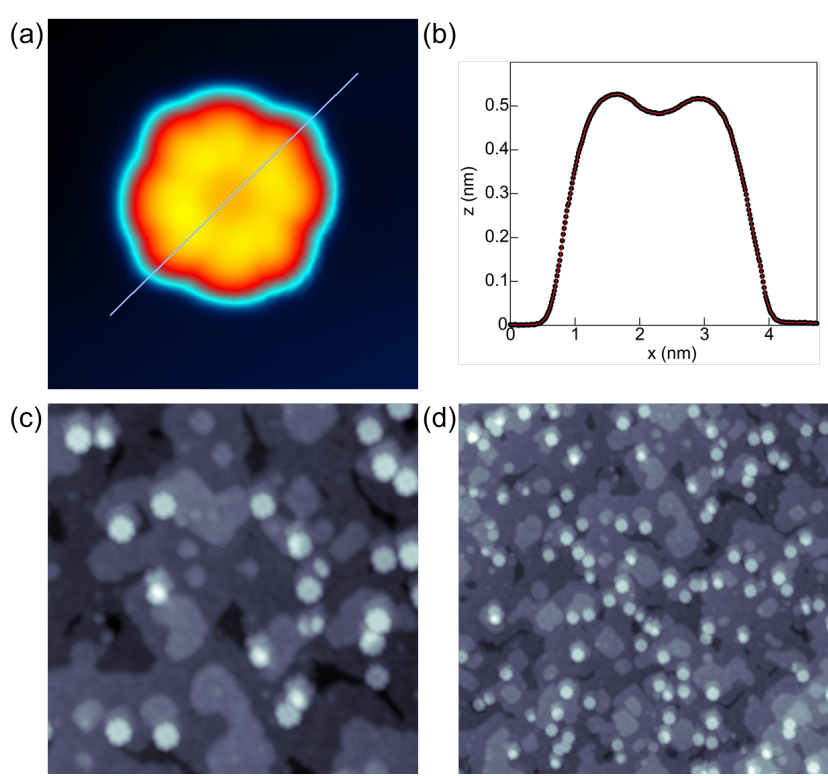

Figure 5: (a) STM image of a single $\mathrm{Tb}_{2} \mathrm{Pc}_{3}$ molecule adsorbed on $\mathrm{Ag}(100)$. Image size $5 \times 5 \mathrm{~nm}^{2}$. (b) Line profile along the cut shown in (a). (c) and (d) $\mathrm{Tb}_{2} \mathrm{Pc}_{3}$ molecules adsorbed on $12 \mathrm{ML} \mathrm{Ni} / \mathrm{Ag}(100)$. Image size $40.5 \times 40.5 \mathrm{~nm}^{2}$ (c) and $81 \times 81 \mathrm{~nm}^{2}$ (d). All images recorded at $4.8 \mathrm{~K}$ in a cryogenic STM.

tive loops taken with opposite photon polarization. It is thus immune to the drift of the photon energy, which sometimes affects fixed-energy measurements of the XMCD intensity, as well as to training effects of the magnetization, which can take place in AFM films. For reasons of speed, the first method was preferred for $\mathrm{MnPc} / \mathrm{Ni}, \mathrm{MnPc} / \mathrm{CoO}, \mathrm{TbPc}_{2} / \mathrm{Ni}$, and $\mathrm{Tb}_{2} \mathrm{Pc}_{3} / \mathrm{Ni}$. Because of its accuracy and the need to accurately measure small vertical shifts due to exchange bias, the second method was preferred for $\mathrm{Tb}_{2} \mathrm{Pc}_{3} / \mathrm{CoO}$ and $\mathrm{Tb}_{2} \mathrm{Pc}_{3} / \mathrm{Mn}$. Finally, we note that throughout this work the XMCD intensity is presented in units of the average XAS intensity, $\left(I^{+}+I^{-}\right) / 2$, which makes it proportional to the atomic magnetic moment of the element under investigation and makes it independent of geometry- and fieldinduced effects on the TEY.

\section{Molecule adsorption on FM and AFM films}

Figure 3 shows four STM images corresponding to (a) $\mathrm{MnPc}$ deposited on $\mathrm{Ag}(100)$, (b) $\mathrm{TbPc}_{2}$ on $13 \mathrm{ML} \mathrm{Ni} / \mathrm{Cu}(100)$, (c) $\mathrm{TbPc}_{2}$ on $3 \mathrm{ML} \mathrm{CoO} / \mathrm{Ag}(100)$ (inset), and (d) $\mathrm{TbPc}_{2}$ on $3 \mathrm{ML}$ $\mathrm{Mn} / \mathrm{Ag}(100)$. We observe that $\mathrm{MnPc}$ and $\mathrm{TbPc}_{2}$ adsorb flat on metal as well as on metal-oxide surfaces, in agreement with previous studies on related systems [61, 62]. The FM and AFM films have an rms roughness smaller than $1.8 \AA$ and present an island-like morphology [56, 63]. Although, depending on the coverage, the lateral dimensions of the metal islands can be as small as $10 \mathrm{~nm}$, we find that most molecules adsorb on flat sites, either in the middle of a terrace or near a step edge. The orientation of the molecules is confirmed by the strong natural dichroism measured by linearly polarized x-ray absorption. Figure 4 (a) and (b) show the $\mathrm{x}$-ray absorption spectra of $\mathrm{TbPc}_{2}$ on the $\mathrm{Ni}$ and $\mathrm{O}(2 \times 2) / \mathrm{Ni}$ surfaces measured at the $\mathrm{N} K$-edge with linearly
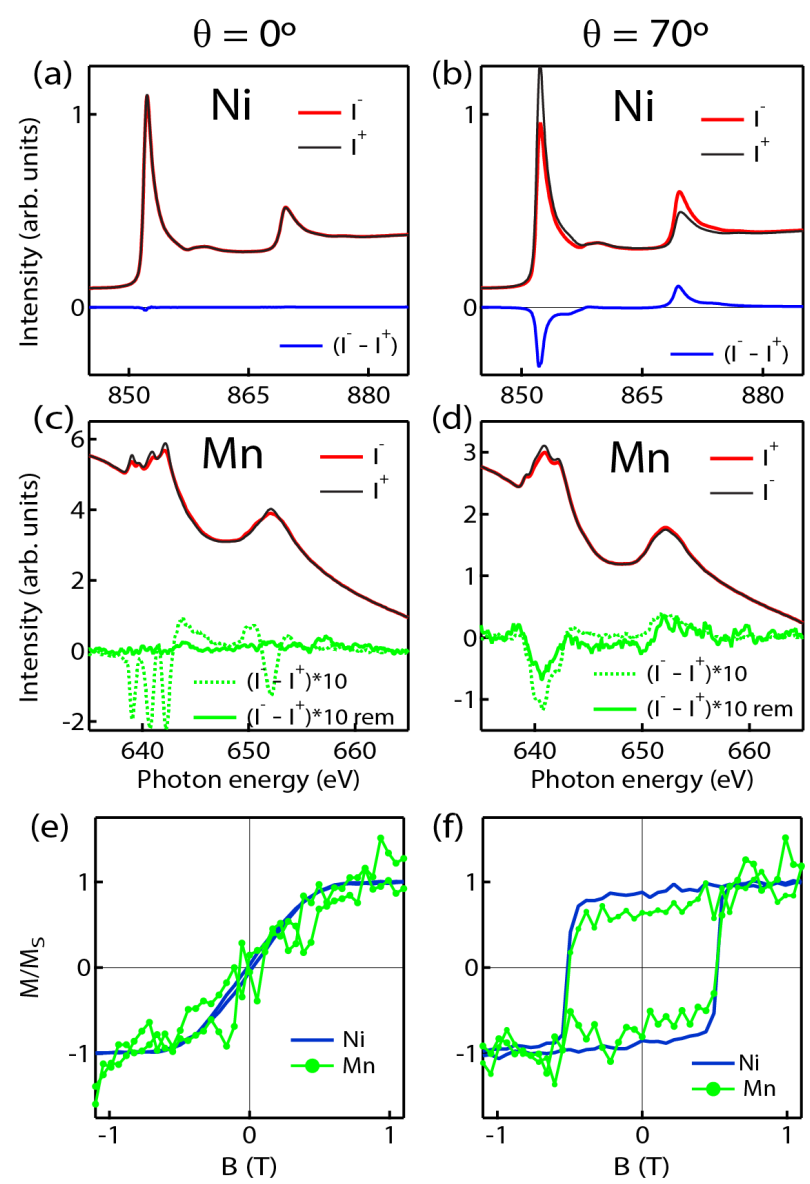

Figure 6: XAS and XMCD spectra of 0.9 ML MnPc/15 ML Ni/Ag(100) recorded at $T=8 \mathrm{~K}$. Ni spectra measured in remanence at (a) $\theta=0^{\circ}$ and (b) $\theta=70^{\circ}$. Mn spectra measured with an applied field of $1.1 \mathrm{~T}$ at (c) $\theta=0^{\circ}$ and (d) $\theta=70^{\circ}$. Element-resolved magnetization loops of $\mathrm{Ni}$ (blue) and $\mathrm{Mn}$ (green) measured at (e) $\theta=0^{\circ}$ and (f) $\theta=70^{\circ}$. The magnetization curves were normalized to the saturation value to compare the shape of the Mn and Ni loops.

polarized light parallel to the surface plane or tilted $70^{\circ}$ out-ofplane. Similarly to Pc, the low energy features between 395 and $405 \mathrm{eV}$ are assigned to $1 s \rightarrow \pi^{*}$ transitions, whereas features above $405 \mathrm{eV}$ are assigned to $1 s \rightarrow \sigma^{*}$ transitions [64]. As the dipole selection rules allow transitions only for the component of electric field vector $(\mathbf{E})$ parallel to the orientation of the $2 p$ like nitrogen orbitals, and the $\pi^{*}$ orbitals are perpendicular to the Pc plane, the larger intensity of the $1 s \rightarrow \pi^{*}$ resonances for out-of-plane $\mathbf{E}$ indicates that most of the $\mathrm{TbPc}_{2}$ molecules lie flat on the surface. We note that in this case strong linear dichroism effects are expected also at the $M_{5}$ edge of $\mathrm{Tb}[46,65]$, as observed in Fig. 4 (c) and (d).

$\mathrm{X}$-ray linear dichroism spectra show that also the $\mathrm{Tb}_{2} \mathrm{Pc}_{3}$ molecules adsorb with the Pc planes parallel to the Ni substrate [Fig. 4 (c)]. As most polynuclear compounds have a limited thermal stability, we checked that the molecules remain intact after evaporation. Figure 5 (a) shows an STM image of $\mathrm{Tb}_{2} \mathrm{Pc}_{3}$ adsorbed on $\operatorname{Ag}(100)$, following sublimation from a crucible heated to $500{ }^{\circ} \mathrm{C}$ on the $\mathrm{Ag}$ substrate held at room temperature. The line profile reported in (b) shows that the apparent height 
of $\mathrm{Tb}_{2} \mathrm{Pc}_{3}$ measured by STM is significantly larger than that of $\mathrm{TbPc}_{2}$ and $\mathrm{TbPc}$ [66]. This, together with the absence of Pc fragments, indicates that the triple-decker Pc complexes remain intact when evaporated on metals. As noted for $\mathrm{TbPc}_{2}$, we find that also $\mathrm{Tb}_{2} \mathrm{Pc}_{3}$ on $\mathrm{Ni}$ adsorbs on planar sites with a preference for island corners, as seen in Fig. 5 (c) and (d). Note that the images of Fig. 5 have been recorded with a cryogenic STM in a quiet environment, whereas those in Fig. 3 have been recorded using the beamline STM shown in Fig. 2, prior to transferring the sample into the XMCD chamber.

\section{Single-decker molecules on ferromagnets: $\mathrm{MnPc} / \mathrm{Ni}$}

The interaction of Pc, OEP, and TTP molecules with FM substrates has been intensively investigated to induce FM behavior in otherwise paramagnetic complexes (see Table I). $\mathrm{MnPc}$ represent an interesting system in this respect, since the $\mathrm{Mn}$ ions carry a large magnetic moment in the pristine complexes [67], which is predicted also to have significant anisotropy [68]. Moreover, density functional calculations and photoemission measurements of $\mathrm{MnPc}$ on Co have shown that the electronic states that form at the molecule-metal interface close to the Fermi level are highly spin-polarized [69], which makes $\mathrm{MnPc} / \mathrm{FM}$ layers of interest as spin filters. The interaction between $\mathrm{MnPc}$ and either Co or Fe surfaces has been shown to be FM [17, 18, 19]. Here we analyze the case of $\mathrm{MnPc} / \mathrm{Ni}$.

Figure 6 shows the XAS and XMCD spectra of a $0.9 \mathrm{ML}$ MnPc / 15 ML Ni film deposited on $\mathrm{Ag}(100)$ measured at a temperature of $8 \mathrm{~K}$. The $\mathrm{Ni}$ spectra are measured in remanence at $\theta=0^{\circ}$ and $\theta=70^{\circ}$ indicate that the $\mathrm{Ni}$ film is magnetized in-plane. Both the XAS and XMCD spectra of Mn, measured in a field of $1.1 \mathrm{~T}$, present a rich multiplet structure with strongly anisotropic lineshape. Such spectra are a fingerprint of the ground state of the $\mathrm{Mn}^{2+}$ ions, which, in analogy with $\mathrm{MnPc} / \mathrm{Ag}(100)$ [70], we assign to a $B_{1 g}$ state with orbital occupation $\left(b_{2 g}\right)^{1}\left(e_{g}\right)^{2}\left(a_{1 g}\right)^{2}$ and intermediate spin $S=3 / 2$. The fact that the XMCD/XAS intensity ratio is quite small for this field and temperature, suggests that charge transfer and/or screening effects induced by the substrate significantly reduce the effective Mn magnetic moment compared to that expected of an isolated molecule.

The XMCD magnetization curves reported in Fig. 6 (e, f) recorded at the $\mathrm{Ni}$ and $\mathrm{Mn} L_{3}$ edges at normal and grazing incidence reveal that the Mn magnetic moment is strongly FMcoupled to Ni. The sign of the exchange interaction is the same as that calculated for Mn impurities in a FM Ni matrix [71], which can be attributed to the direct exchange interaction between the Mn and Ni $3 d$ states, similarly to MnPc/Co [18] and $\mathrm{MnPc} / \mathrm{Fe}$ [72]. As observed for all planar complexes deposited on FM metal surfaces (Table I), the MnPc magnetic moment follows at all fields the magnetization of $\mathrm{Ni}$, adopting the magnetic anisotropy and coercivity of the FM substrate. Under most practical aspects, this means that the molecule and substrate form a single magnetic entity, with a stable molecular magnetic moment but no possibility to independently control the magnetization of one layer with respect to the other.

\section{Double-decker molecules on ferromagnets: $\mathrm{TbPc}_{2} / \mathrm{Ni}$}

$\mathrm{TbPc}_{2}$ is the most representative and studied molecule of the bis(phthalocyaninato) lanthanide family and a prototype singleion SMM [44, 73]. The core of the molecule is a $\mathrm{Tb}^{3+}$ ion sandwiched between two Pc ligands in an eightfold coordinated square antiprism ("double-decker") configuration [Fig. 1 (b)]. The Tb ion has a $4 f^{8}$ electronic configuration with spin and orbital moments equal to $S=3$ and $L=3$, respectively. The strong spin-orbit coupling of the $4 f$ electrons leads to a ground state term with total angular momentum $J=6$, which is further split by the uniaxial ligand field due to the Pc ligands into widely spaced $J_{z}$ substates. The lowest doublet with $J_{z}= \pm 6$ is split by about $54 \mathrm{meV}$ from the $J_{z}= \pm 5$ substates [74], yielding a very large uniaxial magnetic anisotropy and small transition probability between the lowest substates. As a result, $\mathrm{TbPc}_{2}$ shows magnetization hysteresis at low temperature [73]. Ac magnetic susceptibility measurements of $\mathrm{TbPc}_{2}$ diluted in a diamagnetic matrix yield magnetic relaxation times of the order of $0.16 \mathrm{~ms}$ at $40 \mathrm{~K}$ and $1.6 \mathrm{~s}$ at $8 \mathrm{~K}$ [75], whereas XMCD measurements of $\mathrm{TbPc}_{2}$ adsorbed on conducting substrates show magnetic hysteresis only below about $7 \mathrm{~K}$ [46, 47, 76].

Increasing the stability of the molecular magnetic moment is thus a challenge for SMM as well as for simpler paramagnetic molecules. In principle, the same strategy of depositing the molecules onto FM substrates can be applied to SMM, as exemplified in the previous section. SMM, however, including $\mathrm{TbPc}_{2}$, have a larger and more complex structure compared to the planar phthalocyanine and porphyrine derivatives. Moreover, the magnetic ions are separated from the FM substrate by one or more organic ligands. This makes coupling more challenging, but at the same time more intriguing, especially in molecules that present an unpaired $\pi$ electron delocalized over the organic ligands, as is the case for neutral $\mathrm{TbPc}_{2}$ [77].

In this section we analyze the coupling of $\mathrm{TbPc}_{2}$ to $\mathrm{Ni}$ films that have either out-of-plane or in-plane magnetic anisotropy. In the first case, the molecule and substrate easy axes are parallel (Fig. 7), whereas in the second case they are perpendicular to each other (Fig. 8).

\section{1. $\mathrm{TbPc}_{2}$ on Ni films with out-of-plane magnetization}

Figure 7 shows the magnetization of $\mathrm{Tb}\left(\mathbf{M}_{\mathrm{Tb}}\right.$, red curve) and $\mathrm{Ni}\left(\mathbf{M}_{\mathrm{Ni}}\right.$, blue curve) for $0.05 \pm 0.02 \mathrm{ML} \mathrm{TbPc}_{2}$ on $13 \mathrm{ML}$ $\mathrm{Ni} / \mathrm{Cu}(100)$ measured at $8 \mathrm{~K}$. We observe several distinctive features compared to single-decker molecules. First, $\mathbf{M}_{\mathrm{Tb}}$ is coupled antiparallel to $\mathbf{M}_{\mathrm{Ni}}$ at remanence. Second, $\mathbf{M}_{\mathrm{Tb}}$ closely follows $\mathbf{M}_{\mathrm{Ni}}$ only for $|B|<0.1 \mathrm{~T}$. At higher fields, $\mathbf{M}_{\mathrm{Tb}}$ rotates away from $\mathbf{M}_{\mathrm{Ni}}$ and tends to align parallel to $\mathbf{B}$. This occurs as the Zeeman energy gradually increases and finally overcomes the AFM exchange coupling to the substrate, leading to a configuration where $\mathbf{M}_{\mathrm{Tb}}$ and $\mathbf{M}_{\mathrm{Ni}}$ are parallel. Note also that $\mathbf{M}_{\mathrm{Tb}}$ presents a square hysteresis rather than the butterfly loop with near-zero remanence typical of $\mathrm{TbPc}_{2}$ in bulk crystals and nonmagnetic substrates $[47,76,78]$.

The sign of the coupling is opposite to that of $\mathrm{MnPc} / \mathrm{Ni}$ and single-decker phthalocyanine and porphyrine complexes adsorbed on bare FM surfaces, as shown in Table I. This is 


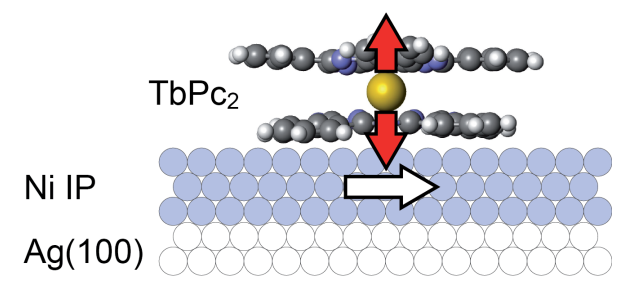

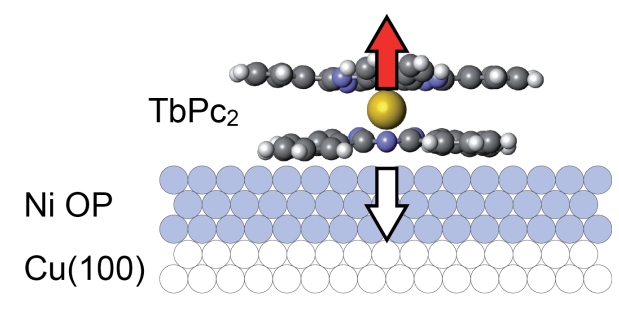
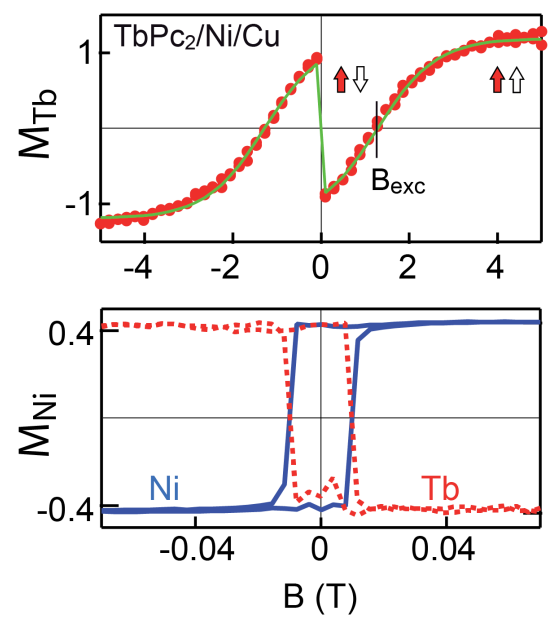

Figure 7: Element-resolved hysteresis loops of $\mathrm{Tb}$ (top panel) and $\mathrm{Ni}$ (bottom panel) for $\mathrm{TbPc}_{2}$ on a $13 \mathrm{ML} \mathrm{Ni}$ film with perpendicular magnetic anisotropy, measured at $T=8 \mathrm{~K}$ at $\theta=0^{\circ}$. The magnetic field is applied perpendicular to the surface. The units of $\mathbf{M}_{\mathrm{Ni}}$ and $\mathbf{M}_{\mathrm{Tb}}$ correspond to the XMCD/XAS ratio at the $L_{3}$ and $M_{5}$ absorption edges. The solid line superposed to $\mathbf{M}_{\mathrm{Tb}}$ is a fit according to Eq. (1). The dashed line in the bottom panel shows the $\mathrm{Tb}$ magnetization at low field normalized to $\mathbf{M}_{\mathrm{Ni}}$. Adapted from Ref. [15].
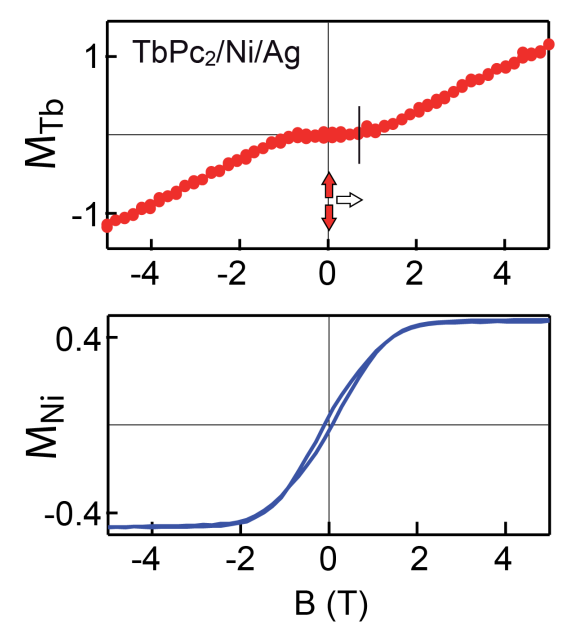

Figure 8: Element-resolved hysteresis loops of $\mathrm{Tb}$ (top panel) and $\mathrm{Ni}$ (bottom panel) for $\mathrm{TbPc}_{2}$ on a $6 \mathrm{ML} \mathrm{Ni}$ film with in-plane magnetic anisotropy, measured at $T=8 \mathrm{~K}$ at $\theta=0^{\circ}$. The magnetic field is applied perpendicular to the surface. Adapted from Ref. [15].

attributed to a Pc-mediated superexchange mechanism, which most likely involves the four $\mathrm{N}$ atoms situated between $\mathrm{Tb}$ and the surface. As the Tb ion does not bind to the substrate, direct exchange coupling between $\mathrm{Tb}$ and $\mathrm{Ni}$ is excluded. Moreover, contrary to $\mathrm{MnPc}, \mathrm{TbPc}_{2}$ retains its intrinsic axial magnetic anisotropy, which strongly favors the out-of-plane direction of the $\mathrm{Tb}$ moment [15]. The competition between the intrinsic SMM properties of $\mathrm{TbPc}_{2}$ and AFM exchange to the substrate can be described using the following Hamiltonian:

$$
H=\mu_{B}(\mathbf{L}+2 \mathbf{S}) \cdot \mathbf{B}-\lambda \mathbf{L} \cdot \mathbf{S}+V_{C F}+\hat{\mathbf{M}}_{\mathrm{Ni}} \cdot \mathbf{K} \cdot \hat{\mathbf{S}}
$$

where $\mu_{B}$ is the Bohr magneton, $\lambda=212 \mathrm{meV}$ the spin-orbit coupling constant, and $V_{C F}=-B_{2} O_{2}^{0}-B_{4} O_{4}^{0}-B_{6} O_{6}^{0}$ the crystal field potential of the $4 f$ states expressed in terms of the Stevens operators $O_{k}^{m}$. Since $V_{C F}$ is not affected by deposition on metals [46], we use the same coefficients $B_{2}=414, B_{4}=-228$, and $B_{6}=33 \mathrm{~cm}^{-1}$ as for the unperturbed molecule [74]. K represents the superexchange tensor between $\mathrm{Ni}$ and $\mathrm{Tb}$, of which we consider only the diagonal out-of-plane $K^{\perp}$ and in-plane $K^{\|}$ components. Equation (1) is used to calculate the expectation value of $\mathbf{M}_{\mathrm{Tb}}=-\mu_{B}(\langle\mathbf{L}\rangle+2\langle\mathbf{S}\rangle)$ as a function of applied field and temperature and fit the magnetization curve in Fig. 7. For simplicity, the fit is restricted to the bottom $J=L+S=6$ multiplet of $\mathrm{TbPc}_{2}$ [74] and we take $\hat{M}_{\mathrm{Ni}}= \pm 1$ for $B \gtrless 0$. The fit has three free parameters: $K^{\perp}, K^{\|}$, and a multiplicative factor that scales $\mathbf{M}_{\mathrm{Tb}}$ to the XMCD intensity. Such parameters reduce to two when $\theta=0^{\circ}$, as $K^{\|}$plays no role in this case. The fit, shown 
(a)
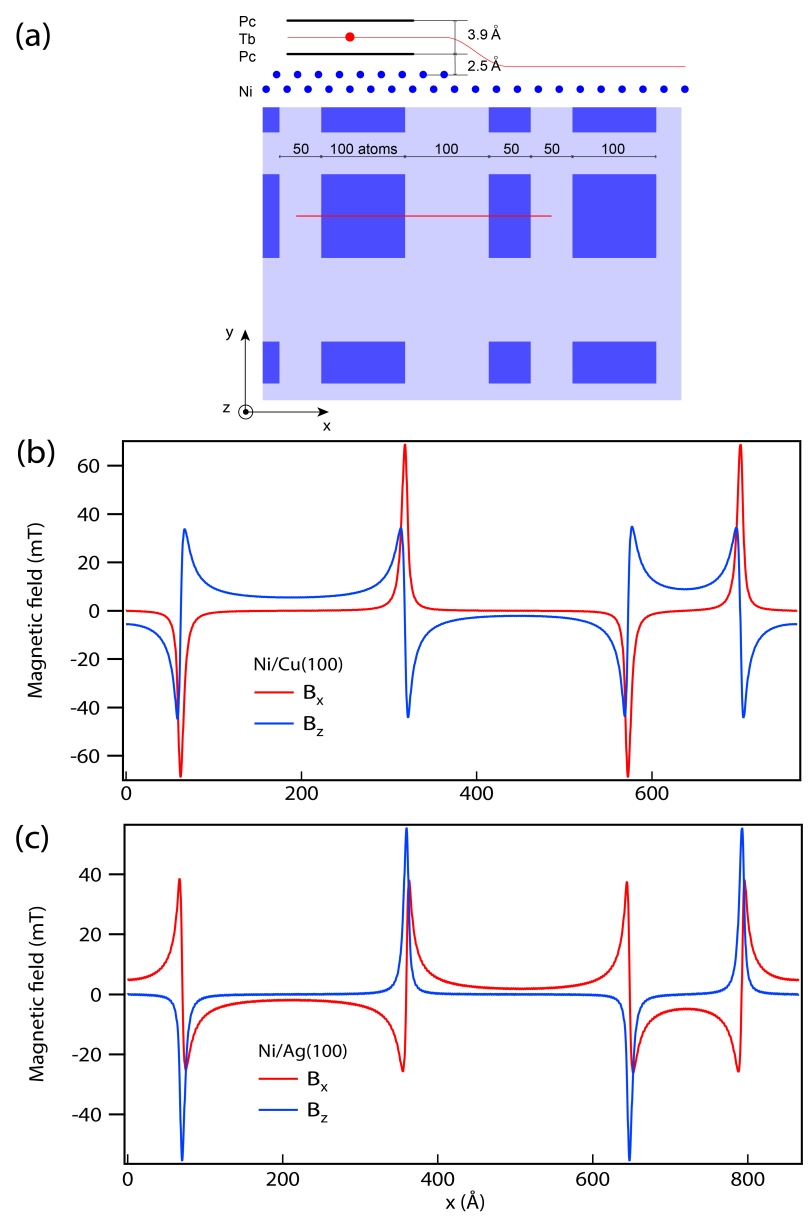

Figure 9: a) Schematic of the Ni surface employed in the calculations of the dipolar field $B_{\text {dip }}$ emanating from the substrate. Dark blue areas indicate second layer $\mathrm{Ni}$ islands, blue dots the position of $\mathrm{Ni}$ atoms. The red line is placed at a distance of $4.45 \AA$ from the topmost Ni plane. (b) $B_{\text {dip }}$ as a function of $x$ calculated along the red line shown in (a) for Ni magnetized out-of-plane . (c) $B_{d i p}$ as a function of $x$ calculated along the red line shown in (a) for $\mathrm{Ni}$ magnetized in-plane.

as a solid green line in Fig. 7, reproduces remarkably well the magnetic behavior of $\mathrm{TbPc}_{2}$, demonstrating that this model captures the main features of the interaction between SMM and the substrate. We obtain $K^{\perp}=-0.67 \pm 0.01 \mathrm{meV}$. Fitting of the hard axis magnetization (not shown, see Ref. [15]) gives $K^{\|}=-14.4 \pm 0.3 \mathrm{meV}$. Such a strong superexchange anisotropy is not unusual for rare earth ions with unquenched $\mathbf{L}$ since the spin-orbit interaction is significant compared to $V_{C F}$ [79]. In such a case, a rotation of the $4 f$ spin implies also a rotation of the electrons' orbit, thereby changing the electronic overlap with neighboring orbitals that is at the origin of superexchange. Anisotropic coupling, moreover, may be a general feature of hybrid metal-organic interfaces, as it has been reported recently also for $\mathrm{Cu}$ porphyrin molecules adsorbed on magnetite [32].

\section{2. $\mathrm{TbPc}_{2}$ on Ni films with in-plane magnetization}

The description of the $\mathrm{Tb}-\mathrm{Ni}$ coupling is more complicate when the substrate has in-plane magnetic anisotropy. Figure 8 shows $\mathbf{M}_{\mathrm{Tb}}$ (red curve) and $\mathbf{M}_{\mathrm{Ni}}$ (blue curve) for $0.05 \mathrm{ML} \mathrm{TbPc}_{2}$ on $6 \mathrm{ML} \mathrm{Ni} / \mathrm{Ag}(100)$. The curves are measured at $8 \mathrm{~K}$ with the external field applied out-of-plane, parallel to the $\mathrm{Tb}$ easy axis. We observe that the coupling between $\mathrm{TbPc}_{2}$ and $\mathrm{Ni}$ remains AFM, as expected since the chemical nature of the moleculemetal interface does not change. However, the magnetic behavior of $\mathrm{Tb}$ is deeply affected by the magnetic anisotropy of the substrate. When the molecule and substrate anisotropy axes are perpendicular to each other, the system is magnetically frustrated. The $\mathrm{Tb}$ axial anisotropy is so strong that only the up and down direction are energetically favored, similar to an Ising system. Therefore, when the substrate is magnetized in-plane, the exchange field mixes in equal amount the up and down $\mathrm{Tb}$ states, leading to zero remanence. In principle, such a behavior can be also modelled by Eq. (1), but for the fact that the experimental $\mathbf{M}_{\mathrm{Tb}}$ continues to increase even after $\mathbf{M}_{\mathrm{Ni}}$ has saturated, whereas $\mathbf{M}_{\mathrm{Tb}}$ calculated using Eq. (1) would also saturate. This discrepancy is still an open issue, but may be ultimately related to local changes of the $\mathrm{Ni}$ magnetic anisotropy induced by the adsorption of $\mathrm{TbPc}_{2}$ that cannot be probed by a spatial averaging technique such as XMCD. In any case, the behavior of $\mathbf{M}_{\mathrm{Tb}}$ up to $B= \pm 0.7$ T can be qualitatively explained by considering that, as long as the out-of-plane component of $\mathbf{M}_{\mathrm{Ni}}$ increases linearly with field, the out-of-plane AFM exchange term $K^{\perp} \hat{M}_{\mathrm{Ni}}$ approximately compensates the Zeeman energy $\mu_{B}(\mathbf{L}+2 \mathbf{S}) B$, leading to $M_{\mathrm{Tb}} \approx 0$.

Our results show that the substrate and molecule magnetic anisotropy axes must be parallel in order to induce magnetic remanence in the SMM. When this is the case, the stability of the $\mathrm{Tb}$ magnetic moment is greatly enhanced compared to $\mathrm{TbPc}_{2}$ in bulk specimens as well as on nonmagnetic substrates $[46,47,76]$. At temperatures above a few degrees $\mathrm{K}$ and timescales larger than a few s, thermal fluctuations and resonant quantum tunnelling between the lowest $J_{z}= \pm 6$ states and between hyperfine levels [78] lead to vanishing remanence for $\mathrm{TbPc}_{2}$. Here, the timescale of the XMCD measurements is of the order $10^{3} \mathrm{~s}$, i.e., the time required to record an hysteresis loop. At $8 \mathrm{~K}$, we find that the value of $\mathbf{M}_{\mathrm{Tb}}$ at remanence is about $80 \%$ of that at $5 \mathrm{~T}$ (Fig. 7), in agreement with the thermally averaged moment expected from Eq. 1. At $80 \mathrm{~K}$ the remanence has reduced to about $10 \%$ of the saturation magnetization, while a finite XMCD intensity is observed up to $100 \mathrm{~K}$ [15]. The thermal stability of $\mathrm{TbPc}_{2}$ can be increased even further, in principle, on substrates with stronger exchange coupling, such as $\mathrm{Co}$ and $\mathrm{Fe}$ films with perpendicular magnetic anisotropy.

\subsection{Magnitude of dipolar field from the substrate}

Besides modelling the exchange field induced by the substrate, we studied the influence of the dipolar field emanating from the Ni surface atoms on the $\mathrm{Tb}$ magnetic moment. Although the dipolar field produced by a continuous magnetization density with either out-of-plane or in-plane orientation is zero outside an infinite surface plane, the discrete atomic structure of a real surface gives rise to finite dipolar fields close to the surface [80]. In order to address this point, we have carried out a model calculation of the dipolar field produced by a (100) Ni surface layer made of 2100 by 2100 atoms. The 
(a) $\quad \mathrm{TbPc}_{2} / \mathrm{O}(2 \times 2) / \mathrm{Ni} / \mathrm{Cu}$
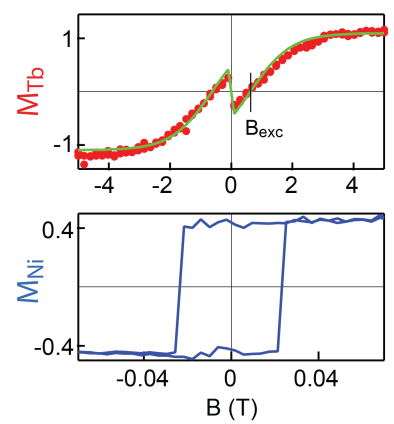

(b)
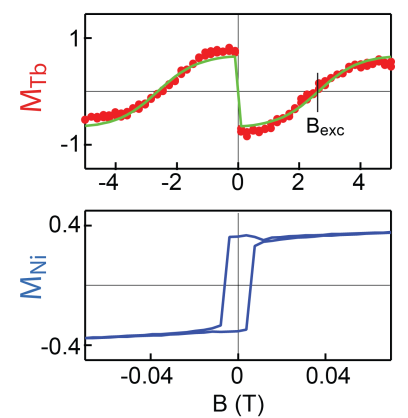

Figure 10: Element-resolved hysteresis loops of $\mathrm{Tb}$ (top) and $\mathrm{Ni}$ (bottom) for (a) $\mathrm{TbPc}_{2} / \mathrm{O} / \mathrm{Ni} / \mathrm{Cu}(100)$ and (b) Li-doped $\mathrm{TbPc}_{2} / \mathrm{Ni} / \mathrm{Cu}(100)$ measured at $\theta=$ $0^{\circ}$ at $T=8 \mathrm{~K}$. The units of $\mathbf{M}_{\mathrm{Ni}}$ and $\mathbf{M}_{\mathrm{Tb}}$ correspond to the XMCD/XAS ratio at the $L_{3}$ and $M_{5}$ absorption edges, respectively. The solid lines superposed to $\mathbf{M}_{\mathrm{Tb}}$ are fits according to Eq. (1). Adapted from Ref. [15].

effects of roughness are included by simulating second layer islands of 10 to $20 \mathrm{~nm}$ lateral size. A diagram of the simulated Ni surface is shown in Fig. 9 (a). A $\mathrm{TbPc}_{2}$ molecule is shown to scale with the Ni lattice constant. The distance between the Pc ligand and the topmost $\mathrm{Ni}$ atoms is taken to be $2.5 \AA$ from density functional calculations of MPc adsorbed on metal surfaces [38], whereas the distance between the bottom and top Pc ligand is taken from Ref. [76]. The number of atoms in the simulation is such that the results can be considered accurate: for instance, adding an additional bottom layer would not change significantly the value of the dipolar field on the surface, due to the exponential decrease of the field with $z$. The calculations were done by adding dipolar fields from individual dipoles, giving a resultant field $\mathbf{B}_{d i p}=\frac{\mu_{0}}{4 \pi} \sum_{i}\left(3\left(\mathbf{m} \cdot \mathbf{r}_{i}\right) \mathbf{r}_{i} / r_{i}^{5}-\mathbf{m} / r_{i}^{3}\right)$, where $\mathbf{r}_{i}$ is the position of the i-th atom, and $\mathbf{m}$ its magnetic moment. For the calculation, we take $m=1 \mu_{B}$ to provide a "worst case" estimate of $B_{d i p}$, given that the magnetic moment of Ni surface atoms is about $0.7 \mu_{B}$. The simulations were performed for $\mathrm{Ni}$ layers with out-of-plane magnetization and $\mathrm{Cu}(100)$ lattice spacing [Fig. 9 (b)] as well as for Ni layers with in-plane magnetization and $\operatorname{Ag}(100)$ lattice spacing [Fig. 9 (c)]. We find that $B_{\text {dip }}$ decreases exponentially with increasing distance from a homogenous Ni layer, reaching values below $0.01 \mathrm{~T}$ for $z>2 \AA$ (not shown). Oscillations of $B_{d i p}$ moving in the $x y$ plane from on-top to hollow sites are already very small at this distance. Figures 9 (b) and (c) show $B_{\text {dip }}$ calculated at $z=4.45 \AA$ above the Ni surface plane, along the red line shown in (a). The effects of roughness are most visible at the edges of the islands, where $B_{\text {dip }}$ attains a maximum value of about $0.07 \mathrm{~T}$ on both surfaces. This is still very small compared to the exchange fields evidenced by our study. Moreover, $B_{d i p}$ changes sign for molecules on top or between islands, resulting in an average field below $0.002 \mathrm{~T}$. Since the XMCD measurements are sensitive to macroscopic surface areas of the order of $0.1 \times 1 \mathrm{~mm}^{2}$, we conclude that dipolar fields can have only a very small influence on the measured magnetic properties of $\mathrm{TbPc}_{2}$ on $\mathrm{Ni}$.

\section{4. $\mathrm{TbPc}_{2}$ on $\mathrm{Li}$ - and $\mathrm{O}$ - modified Ni films}

As the coupling between $\mathbf{M}_{\mathrm{Tb}}$ and $\mathbf{M}_{\mathrm{Ni}}$ is likely mediated by the $\pi$ orbitals of the Pc ligands, it may be possible to increase or decrease the $\mathrm{Tb}-\mathrm{Ni}$ exchange field by modifying the amount of charge transferred between the surface and $\mathrm{TbPc}_{2}$. This can be realized in practice by i) preparing a $(2 \times 2) \mathrm{O}$ buffer layer [63] between $\mathrm{TbPc}_{2}$ and $\mathrm{Ni}$, and ii) doping the $\mathrm{Ni}$ surface with a strong electron donor such as Li. Although we do not control the extent of charge transfer in either case, one can assume that i) leads to oxidation and ii) to a reduction of the Pc ligand. We find that charge depletion induced by $\mathrm{O}$ at the $\mathrm{TbPc}_{2} / \mathrm{Ni}$ interface leads to a reduction of the exchange field, whereas charge donation induced by Li leads to an increase of the exchange field.

The effects of charge transfer on the $\mathrm{Ni}$ magnetization are limited to changes of the coercivity, which we ascribe to modifications of the surface magnetocrystalline anisotropy energy. The magnetic behavior of $\mathrm{TbPc}_{2}$, on the other hand, changes significantly. Figure 10 (a) shows $\mathbf{M}_{\mathrm{Tb}}$ (top) and $\mathbf{M}_{\mathrm{Ni}}$ (bottom) of $\mathrm{TbPc}_{2}$ adsorbed on a $(2 \times 2) \mathrm{O}$ layer prepared on $9 \mathrm{ML}$ $\mathrm{Ni} / \mathrm{Cu}(100)$. The coupling of $\mathrm{Tb}$ to $\mathrm{Ni}$ is substantially reduced compared to $\mathrm{TbPc}_{2} / \mathrm{Ni} / \mathrm{Cu}(100)$. The $\mathrm{Tb}$ remanence is now only $22 \%$ of $\mathbf{M}_{\mathrm{Tb}}$ at $5 \mathrm{~T}$ and the field at which $\mathbf{M}_{\mathrm{Tb}}$ changes sign as the external field exactly compensates the exchange coupling to the substrate, $B=B_{\text {exc }}$, decreases from $1.28 \mathrm{~T}$ on bare Ni to $0.62 \mathrm{~T}$. A fit of $\mathbf{M}_{\mathrm{Tb}}$ according to Eq. (1) reproduces this behavior, giving $K^{\perp}=-0.33 \pm 0.01 \mathrm{meV}$ and $K^{\|}=2 \pm 2 \mathrm{meV}$. The reduction of the absolute value of the exchange energy when $\mathrm{O}$ is intercalated between $\mathrm{TbPc}_{2}$ and $\mathrm{Ni}$ is not unexpected, as it is observed also for planar molecules, e.g., for FeOEP on $\mathrm{O} / \mathrm{Ni}$ relative to bare $\mathrm{Ni}$ [30]. However, in all single phthalocyanine and porphyrin systems investigated to date, $\mathrm{O}$ intercalation leads to a change of sign of the coupling, from FM to $\mathrm{AFM}[19,22,26,30]$. For $\mathrm{TbPc}_{2}$, instead, we find that the out-of-plane coupling remains AFM, whereas the in-plane coupling turns positive but with a large error bar. Competing $\mathrm{FM}$ and AFM interactions have been reported for $\mathrm{Cu}$ porphyrin molecules adsorbed on magnetite [32]. Here, because of the very large axial anisotropy of $\mathbf{M}_{\mathrm{Tb}}$, the magnetic behavior of $\mathrm{TbPc}_{2}$ is mostly sensitive to $K^{\perp}$ rather than $K^{\|}$.

Figure 10 (b) shows $\mathbf{M}_{\mathrm{Tb}}$ (top) and $\mathbf{M}_{\mathrm{Ni}}$ (bottom) of $\mathrm{TbPc}_{2}$ adsorbed on $13 \mathrm{ML} \mathrm{Ni} / \mathrm{Cu}(100)$, after depositing Li from a getter source at $8 \mathrm{~K}$. The amount of $\mathrm{Li}$ is not known precisely, but enough to entirely cover the surface. Opposite to the previous case, we observe that the $\mathrm{Tb}$ remanence is now enhanced, reaching $142 \%$ of $\mathbf{M}_{\mathrm{Tb}}$ at $5 \mathrm{~T}$ and that $B_{\text {exc }}$ has increased up to $2.55 \mathrm{~T}$. The fit of $\mathbf{M}_{\mathrm{Tb}}$ gives $K^{\perp}=-1.37 \pm 0.02 \mathrm{meV}$ and $K^{\|}=-32.8 \pm 0.7 \mathrm{meV}$. We thus find that the exchange coupling field doubles with respect to bare $\mathrm{Ni}$ and quadruples with respect to $\mathrm{O} / \mathrm{Ni}$, reinforcing the AFM alignment between $\mathrm{Tb}$ and $\mathrm{Ni}$. The origin of this effect cannot be explained in detail as neither the charge of $\mathrm{TbPc}_{2}$ on $\mathrm{Ni}$ is known nor the position of the Li dopants. However, we can speculate that adding electrons to the aromatic Pc ligands leads to a more efficient superexchange process. The neutral unsupported $\left[\mathrm{TbPc}_{2}\right]^{0}$ molecule has two spin systems: one strongly localized on the $4 f$ states, with $J=6$, and the other delocalized over the two Pc ligands 

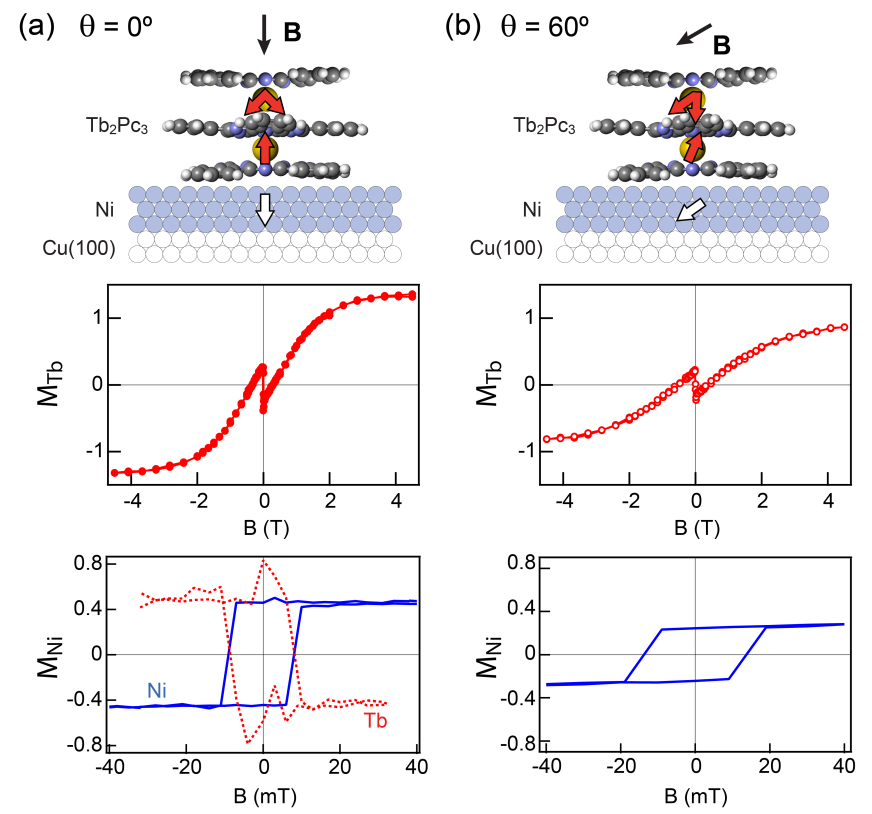

Figure 11: Element-resolved hysteresis loops of $\mathrm{Tb}$ (top) and $\mathrm{Ni}$ (bottom) for $\mathrm{Tb}_{2} \mathrm{Pc}_{3} / 13 \mathrm{ML} \mathrm{Ni} / \mathrm{Cu}(100)$ measured at (a) $\theta=0^{\circ}$ and (b) $\theta=60^{\circ}$ at $T=8 \mathrm{~K}$. The units of $\mathbf{M}_{\mathrm{Ni}}$ and $\mathbf{M}_{\mathrm{Tb}}$ correspond to the XMCD/XAS ratio at the $L_{3}$ and $M_{5}$ absorption edges, respectively. The dashed line in the bottom panel of (a) shows $\mathbf{M}_{\mathrm{Tb}}$ at low field normalized to $\mathbf{M}_{\mathrm{Ni}}$.

due to an unpaired $\pi$ electron with spin $S=1 / 2$ [77]. Electron doping of MPc by alkali metals has been shown to induce siteselective filling of molecular orbitals, including the aromatic $\pi$-states delocalized over the Pc ligand [81], as well as changes of the spin of the metal centers and ligand field [70]. For $\mathrm{YPc}_{2}$ adsorbed on $\mathrm{Au}(111)$ it has been found that $\mathrm{Cs}$ doping leads to the filling of a $\pi$ orbital of the Pc ligand $[82,83]$. Because Ni is a more reactive substrate than $\mathrm{Au}$, we expect the lowest unoccupied $\pi$ orbital of $\mathrm{TbPc}_{2}$ to be partially filled on bare Ni, almost unfilled on the O-covered surface, and completely filled for the Li-doped complexes. Since superexchange tends to favor FM coupling between two magnetic atoms if the intervening negative ion has a singly occupied orbital and AFM coupling for a doubly occupied one, this hypothesis would explain why AFM coupling is so much weaker for $\mathrm{TbPc}_{2} /(2 \times 2) \mathrm{O} / \mathrm{Ni}$ compared to $\mathrm{Li} / \mathrm{TbPc}_{2} / \mathrm{Ni}$. More precise knowledge on the charging of the $\mathrm{Pc}$ orbitals and their energy position relative to the $\mathrm{Ni}$ and $\mathrm{Tb}$ states, however, is required to reach a definitive conclusion on this point.

\section{Triple-decker molecules on ferromagnets: $\mathrm{Tb}_{2} \mathrm{Pc}_{3} / \mathrm{Ni}$}

Pc and porphyrin derivatives with double- or triple-decker structure have been investigated for a long time due to their intrinsic semiconducting properties [84], multiple redox activity [85], and SMM behavior [86, 87], which are of interest to realize field-effect transistors [88, 89] and model information storage media [90]. In the triple-decker complexes, the lanthanide ions are placed along the fourfold symmetry axis at a distance of about $3.6 \AA$ [Fig. 1 (c)]. The presence of two $4 f$ systems, each behaving as a SMM [86, 87], and of delocalized $\pi$ electrons provides the opportunity to study coupling effects with magnetic interfaces as well as at the intramolecular level. In the pristine compounds, the interaction between the lanthanide magnetic moments was determined to be of dipolar character, with negligible contribution from exchange [86, 91]. This interaction, which favors the parallel alignment of the magnetic moments along the easy axis, becomes apparent in magnetic susceptibility measurements of $\mathrm{Tb}_{2} \mathrm{Pc}_{3}$ at temperatures below $10 \mathrm{~K}$ [91].

In order to study the magnetic behavior of $\mathrm{Tb}_{2} \mathrm{Pc}_{3}$ on $\mathrm{FM}$ substrates, we prepared Ni films with out-of-plane magnetization on $\mathrm{Cu}(100)$, analogously to the study of $\mathrm{TbPc}_{2}$ reported in Sect. 5. Figure 11 shows the element-resolved magnetization of $\mathrm{Tb}_{2} \mathrm{Pc}_{3} / 13 \mathrm{ML} \mathrm{Ni} / \mathrm{Cu}(100)$ measured by XMCD at $T=8 \mathrm{~K}$. Here, $\mathbf{M}_{\mathrm{Tb}}$ represents the $\mathrm{Tb}$ magnetic moment averaged over the top and bottom $\mathrm{Tb}$ ions. We observe that $\mathbf{M}_{\mathrm{Tb}}$ is coupled antiparallel to $\mathbf{M}_{\mathrm{Ni}}$ at remanence, and it rotates parallel to the external field when $|B|>B_{\text {exc }}=0.3 \mathrm{~T}$. The magnetic moments of $\mathrm{Tb}$ and $\mathrm{Ni}$ at saturation, estimated by the XMCD asymmetry $\left(I^{+}-I^{-}\right) /\left[\left(I^{+}+I^{-}\right) / 2\right]$, are consistent with those measured in the double-decker samples. This behavior is qualitatively similar to that of $\mathrm{TbPc}_{2} / \mathrm{Ni} / \mathrm{Cu}(100)$ in Fig. 7. However, the remanence value of $\mathbf{M}_{\mathrm{Tb}}$ is only $20 \%$ of the saturation magnetic moment compared to $80 \%$, and $B_{\text {exc }}$ is about one fourth of that measured for $\mathrm{TbPc}_{2}$. We therefore conclude that only the bottom $\mathrm{Tb}$ ion is AFM coupled to $\mathrm{Ni}$, whereas the top $\mathrm{Tb}$ ion is essentially unperturbed by the FM substrate. The reduced remanence is consistent with the the observation that the two $\mathrm{Tb}$ ions in $\mathrm{Tb}_{2} \mathrm{Pc}_{3}$ relax independently of each other [86]. The small value of $B_{\text {exc }}$ compared to $\mathrm{TbPc}_{2}$ is consistent with $\mathbf{M}_{\mathrm{Tb}}$ being the average of two curves, one with $B_{\text {exc }}=0$ and the other with $B_{\text {exc }} \neq 0$. Moreover, the dipolar field produced by one $\mathrm{Tb}$ ion on the other reduces $B_{\text {exc }}$ even further, as the top magnetic moment tends to pull the bottom one towards the direction of the external field. Based on their distance, we estimate that the dipolar field is of the order of $0.25 \mathrm{~T}(0.12 \mathrm{~T})$ at the position of atom $\mathrm{A}$ when the magnetic moment of atom B points out-of-plane (in-plane). These results show that the length scale of exchange coupling induced by a FM substrate is very small and that, in polynuclear SMM, fluctuations of the ionic spins not coupled to the FM may reduce the overall stability of the molecular magnetic moment.

\section{Exchange bias on antiferromagnetic substrates}

Most studies of molecules on magnetic substrates have been carried out on FM surfaces. In such a case, the magnetization of the substrate is orders of magnitude larger compared to that of a molecular layer. Moreover, for planar molecules, the magnetic moment is rigidly coupled to the substrate magnetization. It is therefore impossible to manipulate the molecule magnetic moment independently from the substrate. One may wonder if there are alternative ways to stabilize and control the magnetic behavior of paramagnetic molecules and SMM using substrates with no net magnetization. This may be achieved, in principle, by coupling the molecules to an AFM surface, in analogy with exchange-biased FM/AFM thin films [11]. There are, however, 
several issues that make this goal a difficult one. 1) Exchange bias is triggered locally by the presence of sparse pinned uncompensated spins in the AFM $[92,93,94]$; in FM/AFM films the bias extends to the whole FM layer because the system is structurally and magnetically continuous. Molecules, on the other hand, are discrete magnetic elements. There is no mechanism guaranteeing that a single molecule will be exchangebiased, unless this adsorbs on or creates a pinning site. 2) Even if this occurs, exchange bias is unlikely to extend to all the molecules on the surface unless some kind of intramolecular coupling exists, which is usually not the case [95]. 3) Finally, exchange bias requires the polarization of the pinned uncompensated spins of the AFM during field cooling (FC), which is usually achieved due to the proximity of the FM layer. As thermal fluctuations tend to randomize the orientation of the molecular magnetic moment at temperatures above a few degrees $\mathrm{K}$, well below the typical ordering temperature of AFM layers, the pinned spins in the AFM may not align along the FC direction. These three problems need to be addressed in order to establish if and how single molecules and molecular layers can be exchanged biased by an AFM substrate.

Here we report a series of experiments aimed at establishing the presence or absence of molecular exchange bias at the interface between metal-organic complexes and different types of AFM substrates, namely insulating $\mathrm{CoO}$ and metallic $\mathrm{Mn}$ thin films deposited on $\mathrm{Ag}(100)$ [16]. We chose $\mathrm{CoO}$ and $\mathrm{Mn}$ to compare the behavior of oxide and metal AFM. CoO is a model type II insulating AFM that grows epitaxially on $\mathrm{Ag}(100)$. It has a large magnetocrystalline anisotropy, which favors large exchange bias $\left(H_{E}\right)$ and coercive fields $\left(H_{C}\right)$ in FM/AFM bilayers. Moreover, both the Néel temperature $\left(T_{N}\right)$ and the magnetic anisotropy of $\mathrm{CoO}$ thin films can be controlled by epitaxy $[96,97]$, which makes this system particularly interesting for the investigation of molecular exchange bias phenomena. Elemental Mn thin films grown on $\operatorname{Ag}(100)$ [98, 99, 100] were preferred over other types of metallic AFM, such as NiMn and IrMn, in order to simplify the sample preparation procedure and avoid the presence of different structural and magnetic phases that appear in bimetallic AFM alloys as a function of composition and thickness [101]. Mn layers grow epitaxially on single crystal $\mathrm{Ag}(100)$ forming a two-layer thick superficial alloy at room temperature, which is continued by an almost pure $\mathrm{Mn}$ phase with bct structure above the third layer [100, 102]. It is known that $\mathrm{Mn}$ thin films grown on $\mathrm{Ag}(100)$ present large local magnetic moments and AFM order [99, 103, 104], with predicted $c(2 \times 2)$ magnetic unit cell $[104,105]$.

\section{1. $\mathrm{TbPc}_{2}$ and $\mathrm{MnPc}$ on $\mathrm{CoO}$}

We present first the results obtained for $\mathrm{TbPc}_{2}$ deposited on a $10 \mathrm{ML}$ thick $\mathrm{CoO}$ layer, $\mathrm{FC}$ from 300 to $8 \mathrm{~K}$ in a field $B=+5 \mathrm{~T}$ applied perpendicular to the surface (Fig. 12). The CoO substrate presents a very weak field-induced XMCD at the $L_{2,3}$ edges of Co [Fig. 12 (a)], as expected for a nominally compensated AFM surface. The nonzero XMCD is attributed to the presence of rotatable uncompensated Co spins polarized by the external field. The $\mathrm{TbPc}_{2}$ molecules, on the other hand, present
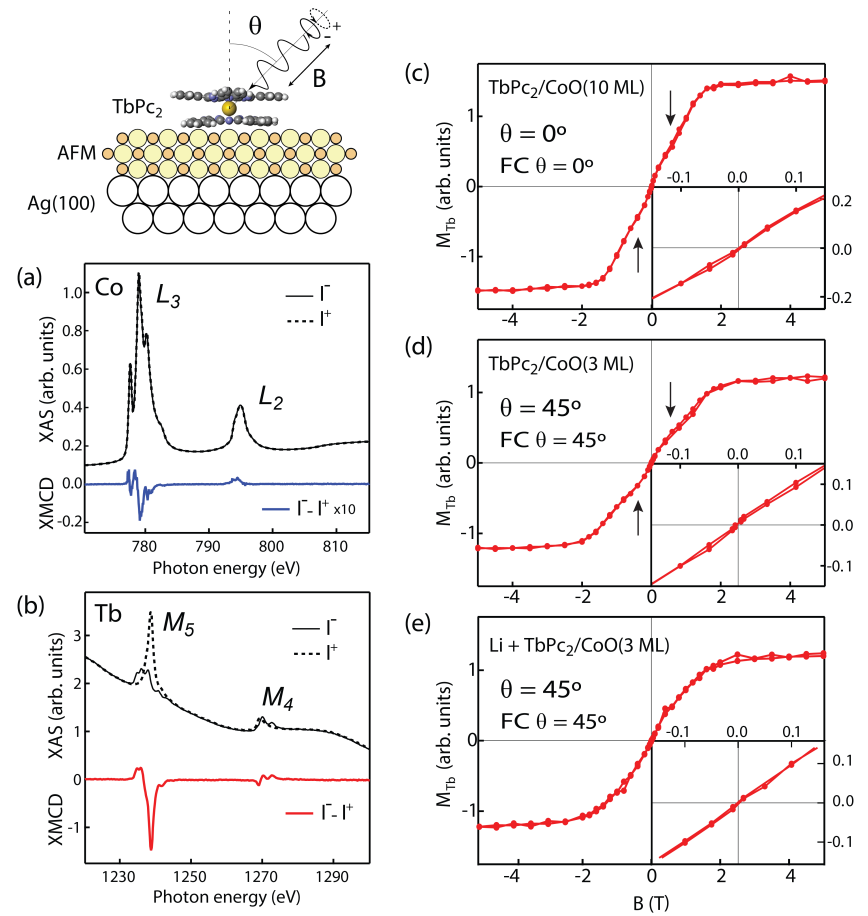

Figure 12: XAS and XMCD spectra of $\mathrm{TbPc}_{2} / \mathrm{CoO}(10 \mathrm{ML}) / \mathrm{Ag}$ recorded the $L_{2,3} \mathrm{Co}$ (a) and $M_{4,5} \mathrm{~Tb}(\mathrm{~b})$ edges after FC at $B=5 \mathrm{~T}, \theta=0^{\circ}$. (c) Magnetization loop of $\mathrm{TbPc}_{2}$ deposited on the $10 \mathrm{ML} \mathrm{CoO}$ film after FC, recorded at $\theta=0^{\circ}$. (d) Magnetization loop of $\mathrm{TbPc}_{2}$ deposited on a $3 \mathrm{ML} \mathrm{CoO}$ film after FC at $\theta=45^{\circ}$, measured at the same angle. The arrows indicate inflection points during a field sweep. (e) Magnetization loop of the sample shown in (d) after Li deposition. Insets: Details of the low field region. Units refer to the intensity ratio $2\left(I^{+}-I^{-}\right) /\left(I^{+}+I^{-}\right)$measured at the $M_{5}$ Tb edge. Inset: Detail of the low field region. Units refer to the intensity ratio $2\left(I^{+}-I^{-}\right) /\left(I^{+}+I^{-}\right)$measured at the $M_{5} \mathrm{~Tb}$ edge. All measurements have been carried out at $T=8 \mathrm{~K}$. Adapted from Ref. [16]. 
a very large XMCD signal [Fig. 12 (b)], which is fully saturated at $5 \mathrm{~T}$. Figure 12 (c) shows the $\mathrm{Tb}$ magnetization versus applied field after FC. The loop is closed (see inset) and antisymmetric about the origin, indicating that there is no measurable exchange bias in this system. The observed paramagnetic behavior is consistent with that of $\mathrm{TbPc}_{2}$ molecules deposited on nonmagnetic metal substrates, which do not show hysteresis at $T=8 \mathrm{~K}[46,47]$. Differently from $\mathrm{TbPc}_{2}$ deposited on metals, however, we note that the $\mathrm{Tb}$ magnetization is not a smooth curve but presents inflection points at $B= \pm 0.5 \mathrm{~T}$, indicated by arrows in Figure 12 (c) and (d). These features correspond to the plateaus of the "butterfly" hysteresis cycle of $\mathrm{TbPc}_{2}$ measured below the blocking temperature in molecular crystals [78], suggesting that they are related to quantum tunneling effects in molecules that interact weakly with the environment. Interestingly, they disappear upon charge doping, as shown in Fig. 12 (e).

To check whether the absence of exchange bias depends on the fluctuating magnetic moment of $\mathrm{TbPc}_{2}$ during $\mathrm{FC}$, we decreased the thickness of the $\mathrm{CoO}$ layer to $3 \mathrm{ML}$, which is expected to reduce its Néel temperature to about $20 \mathrm{~K}$ [96]. Figure 12 (d) shows the $\mathrm{Tb}$ magnetization of $\mathrm{TbPc}_{2} / \mathrm{CoO}(3 \mathrm{ML}) / \mathrm{Ag}$ after $\mathrm{FC}$ at $B=+5 \mathrm{~T}$ and $\theta=45^{\circ}$. We used this geometry to polarize and probe simultaneously the out-of-plane and in-plane magnetization components since, a priori, it is not known in which direction it is easier to pin the uncompensated spins of $\mathrm{CoO}$. Again, however, we found no indication of exchange bias. A negative result was obtained also after depositing Li on this surface [Fig. 12 (e)] to enhance the exchange coupling between $\mathrm{Co}$ and $\mathrm{Tb}$, in analogy with the results reported in Sect. 5.4. Finally, we considered the possibility that the easy axes of $\mathrm{TbPc}_{2}$ and $\mathrm{CoO}$ are perpendicular to each other and the magnetic anisotropy stronger than Tb-Co exchange, in which case the bias field will have no effect on the $\mathrm{Tb}$ magnetization. This may happen because compressive strain is expected to favor preferential in-plane orientation of the magnetic moments in $\mathrm{CoO} / \mathrm{Ag}(100)$ [97], differently from bulk $\mathrm{CoO}$ where the spins align close to the (111) direction [106]. However, measurements of $\mathrm{TbPc}_{2}$ deposited on a tensile-strained $5 \mathrm{ML}$ thick film of $\mathrm{CoO}$ on $45 \mathrm{ML} \mathrm{MnO} / \mathrm{Ag}(100)$ with outof-plane magnetic anisotropy [97] also provided no evidence of exchange bias [16]. We conclude, therefore, that either the exchange coupling between $\mathrm{TbPc}_{2}$ and $\mathrm{CoO}$ is too weak or the density of pinned uncompensated spins too low to produce sizeable bias effects within the sensitivity of the present study. Measurements of $\mathrm{MnPc} / 15 \mathrm{ML} \mathrm{CoO} / \mathrm{Ag}(100) \mathrm{FC}$ at $\theta=0^{\circ}$ and $70^{\circ}$ (not shown) confirm the absence of exchange bias on $\mathrm{CoO}$ also for single-decker complexes, which suggests that AFM oxides are not suited to bias metal-organic molecules.

\section{2. $\mathrm{TbPc}_{2}$ on $\mathrm{Mn}$}

The measurements reported in Sect. 5.4 show that the exchange coupling between $\mathrm{TbPc}_{2}$ and a FM substrate is larger for bare metal surfaces compared to oxygen-covered surfaces. It is therefore likely that the same holds for AFM metals compared to AFM oxides. We thus deposited $\mathrm{TbPc}_{2}$ on a 3 ML-thick Mn films grown on $\mathrm{Ag}(100)$. The XMCD asymmetry measured on (a)

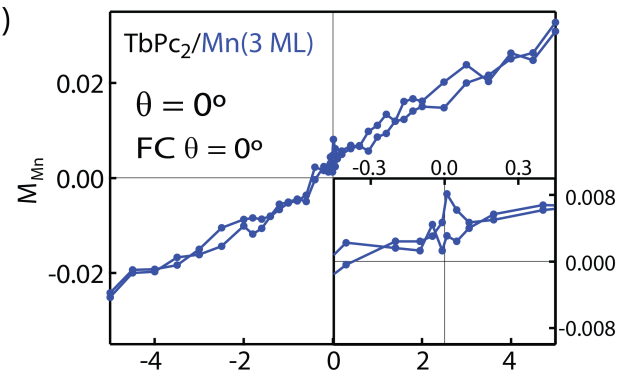

(b)

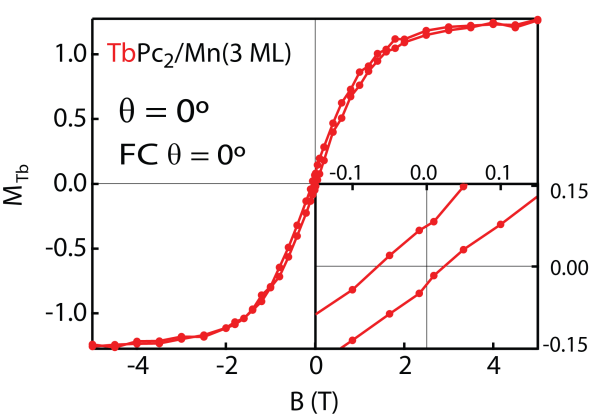

Figure 13: Magnetization loops of $\mathrm{Mn}$ (a) and $\mathrm{Tb}$ (b) measured on $\mathrm{TbPc}_{2} / \mathrm{Mn}(3 \mathrm{ML}) / \mathrm{Ag}(100)$, after FC at $\theta=0^{\circ}$ and $B=5 \mathrm{~T}$, recorded at $\theta=0^{\circ}$ and $T=8 \mathrm{~K}$. Inset: Detail of the low field region. Units refer to the intensity ratio $2\left(I^{+}-I^{-}\right) /\left(I^{+}+I^{-}\right)$measured at the $L_{3}$ Mn edge (a) and $M_{5}$ Tb edge (b). Adapted from Ref. [16].

the Mn films was between 2 and $3 \%$ at $5 \mathrm{~T}$ and $8 \mathrm{~K}$. This is much smaller than the $50 \%$ measured for Mn atoms [107], or the $40 \%$ measured for Mn clusters [108] and NiMn surface alloys [109], showing that our Mn films are indeed AFM. Figure 13 (a) shows the $\mathrm{Mn}$ magnetization $\mathbf{M}_{\mathrm{Mn}}$ measured after FC to $8 \mathrm{~K}$ in an applied field of $5 \mathrm{~T}$ applied perpendicular to the surface. By reducing $B$ from +5 to $+0.05 \mathrm{~T}$, we observe a ten fold reduction of the XMCD intensity. When reversing the field to $B=-0.05 \mathrm{~T}$, the sign of the XMCD remains negative, whereas the XMCD measured at $-5 \mathrm{~T}$ reverses sign but is smaller by $14 \%$ with respect to that measured at $+5 \mathrm{~T}$. This behavior suggests the presence of uncompensated Mn spins, part of which rotate with the field and part pinned parallel to the FC direction. The ratio between the vertical loop shift and the difference $\mathbf{M}_{\mathrm{Mn}}(5 T)-\mathbf{M}_{\mathrm{Tb}}(-5 T)$ indicates that about $(7 \pm 2) \%$ of the uncompensated spins are pinned, while we estimate that the uncompensated spins (pinned and unpinned) are about $(3 \pm 1) \%$ of the total Mn coverage, that is, $0.09 \pm 0.03 \mathrm{ML}$ [16].

Figure 13 (b) shows that the magnetization of $\mathrm{TbPc}_{2}$ on $\mathrm{Mn}$ is hysteretic and exhibits finite remanence and coercivity $H_{C}=44 \pm 4 \mathrm{mT}$. Furthermore, $M_{\mathrm{Tb}}$ is shifted along the field axis by an amount $H_{E}=-22 \pm 4 \mathrm{mT}$. The sign of the shift is consistent with the parallel alignment of the Tb magnetic moment and pinned Mn spins. We also find that both $H_{C}$ and $H_{E}$ decrease significantly for a $\mathrm{TbPc}_{2} / \mathrm{Mn}$ sample $\mathrm{FC}$ at $\theta=90^{\circ}$, perpendicular to the $\mathrm{TbPc}_{2}$ easy axis [16]. These observations are tell-tale signatures of exchange bias in a molecular systems, analogously to FM/AFM films where the shift of the hysteresis loop is often accompanied by an enhanced coercivity [11].

The measurements in Fig. 13 demonstrate that molecular 
complexes can be exchanged-bias by an AFM film, notwithstanding the fact that the molecular magnetic moment fluctuates during FC. As the blocking temperature of $\mathrm{TbPc}_{2}$ on metal substrates, measured on the timescale of XMCD experiments, is about $2 \mathrm{~K}$ [47], below the minimum temperature reached in this study $(8 \mathrm{~K})$, the alignment of the pinned spins must occur in the paramagnetic regime [110] due to the field-induced magnetization of $\mathrm{TbPc}_{2}$. Once AFM order has set in, the uncompensated exchange field from the substrate inhibits the relaxation of the $\mathrm{Tb}$ magnetic moment, giving rise to hysteresis. The remaining question is whether exchange bias may involve an entire molecular layer or only a few sparse molecules. The skewed shape of the loop and the small remanence of $\mathbf{M}_{\mathrm{Tb}}$ indicate that only a small fraction of the $\mathrm{TbPc}_{2}$ molecules is exchange-coupled to the substrate, consistent with the presence of a small percentage of pinned spins. This is very different from the case of $\mathrm{TbPc}_{2}$ deposited on Ni reported in Sect. 7, where the square hysteresis of $M_{\mathrm{Tb}}$ shows that most molecules are coupled to the FM substrate. As XMCD averages over many molecules, pinned and unpinned, we believe that both $H_{E}$ and $H_{C}$ would be much larger if measured on a single pinned molecule.

\section{Conclusions}

In summary, we have investigated the exchange coupling properties of metal-organic molecules to FM and AFM substrates. Most studies of the magnetic interaction between molecules and surfaces focus on planar complexes, such as metal-phthalocyanines and porphyrins. The magnetic moment of these molecules, as shown in Table I, invariably couples parallel to that of FM metal substrates such as Fe, Co, and Ni. Here we reported the case of $\mathrm{MnPc}$ adsorbed on $\mathrm{Ni}$, for which, similar to other systems, we found that the Mn magnetic moment is rigidly coupled to the substrate magnetization. The direction of the magnetic moment, magnetic anisotropy, and coercivity are indistinguishable from the substrate. These systems are evidently of interest as spin filters in planar devices such as magnetic tunnel junctions, but not as carriers of magnetic information.

Additionally, the fabrication of tunnel barriers and magnetic electrodes for spintronic devices may require the use of thicker molecular structures, either to control their electrical conductivity, e.g., by increasing the spacing between metallic contacts, or to augment their total magnetic moment. We therefore investigated the coupling of double-decker and triple-decker complexes to different types of substrates where the relevant interactions are no longer confined to the molecular ligands in contact with a magnetic surface, but extend also in the normal direction away from the interface.

We focused first on $\mathrm{TbPc}_{2}$ as a prototype single-ion SMM, which possesses bi-stable magnetic states at low temperature. We showed that the magnetic moment of $\mathrm{TbPc}_{2}$ deposited on $\mathrm{Ni}$ couples antiparallel to that of the substrate. If the easy magnetization axis of $\mathrm{TbPc}_{2}$, which is out-of-plane, coincides with that of the $\mathrm{Ni}$ film, the magnetic moment of $\mathrm{Tb}$ is effectively stabilized by the interaction with the substrate, resulting in a square magnetization hysteresis curve with nearly saturated magnetic remanence at zero applied field. Finite remanence persists up to $100 \mathrm{~K}$, a temperature that is two orders of magnitude higher compared to the blocking temperature of $\mathrm{TbPc}_{2}$ on nonmagnetic surfaces. Depending on the strength of the applied magnetic field, we observed that both antiparallel and parallel magnetic configurations can be reached, as the Zeeman interaction compensates and eventually overcomes the exchange coupling between $\mathrm{Tb}$ and $\mathrm{Ni}$. This behavior is similar to that of an exchange spring magnet, where a hard magnetic layer stabilizes an exchange-coupled soft layer [10], although the coupling here is AFM rather than FM. If the easy magnetization axis of the $\mathrm{Ni}$ film is orthogonal to that of $\mathrm{TbPc}_{2}$, we observed pronounced frustration effects as the molecule magnetization cannot align with the substrate at equilibrium, which leads to zero remanence at zero field.

Given that $\mathrm{Tb}$ and $\mathrm{Ni}$ are physically separated by a Pc ligand, our data indicate that the coupling between $\mathrm{Tb}$ and $\mathrm{Ni}$ atoms is mediated by superexchange. In agreement with this hypothesis, we find that the strength of the molecule-substrate coupling is smaller compared to single-decker molecules and can be tuned by electron or hole doping of the interface, which is expected to change the occupation of the Pc electron orbitals. This behavior also shows that the interface chemistry and magnetic response are intimately related in these systems.

The triple-decker $\mathrm{Tb}_{2} \mathrm{Pc}_{3}$ molecules deposited on $\mathrm{Ni}$ behave qualitatively similar to $\mathrm{TbPc}_{2}$. However, both the remanence and exchange field are significantly reduced with respect to the double-decker case, consistently with a model where only the bottom $\mathrm{Tb}$ ion is exchange-coupled to the substrate and the top $\mathrm{Tb}$ ion relaxes independently from either. Therefore, exchange coupling does not extend upwards from the FM interface in multiple-decker molecules. Moreover, the thermal stability of the bottom $\mathrm{Tb}$ ion appears to be diminished by the dipolar field generated by the top $\mathrm{Tb}$ ion.

Finally, we investigated the coupling of $\mathrm{TbPc}_{2}$ and $\mathrm{MnPc}$ on AFM substrates. On $\mathrm{CoO}$, independently of the thickness, spin orientation, FC direction, and electron doping of the interface, we found no indication of exchange bias. $\mathrm{TbPc}_{2}$ behaves as an isolated SMM above the blocking temperature, with fully reversible and symmetric magnetization loops. Similar observations for MnPc lead us to conclude that the magnetic coupling on oxide AFM is too weak to be effective for metal-organic molecules. On the other hand, when $\mathrm{TbPc}_{2}$ is deposited on AFM Mn thin films, we find magnetic hysteresis and a negative horizontal shift of the Tb magnetization loop after field cooling, consistent with the observation of pinned spins in the Mn layer coupled parallel to the $\mathrm{Tb}$ magnetic moment. The bias field is found to be maximum when the cooling field is set parallel to the SMM easy axis. From the shape of the $\mathrm{TbPc}_{2}$ magnetization curve, we infer that exchange bias occurs at the level of single molecules. In the future, achieving control over the origin of the pinned spins and positioning of the molecules next to them may result in new applications that exploit the interaction between SMM and AFM, such as spin valves and spin filters, where the molecular magnetic moment is simultaneously stabilized and biased by unidirectional exchange coupling.

In synthesis, exchange coupling to FM and AFM surfaces 
offsets the response of metal-organic molecules to temperature, applied magnetic fields, and electric currents. The effects described in this work can be used to achieve control over the electronic and magnetic properties of molecular complexes deposited on inorganic substrates, e.g., to stabilize the molecular magnetic moments against thermal fluctuations or modify the spin-polarization of magnetic interfaces. In SMM, the competition between substrate-induced exchange coupling, Zeeman interaction, and magnetic anisotropy gives rise to several metastable magnetic configurations that may be used for storing magnetic information.

\section{Acknowledgements}

We acknowledge the ESRF for the provision of beamtime and technical assistance. We received financial support from the European Research Council (StG 203239 NOMAD), Ministerio de Ciencia e Innovación (MAT2010-15659), Agència de Gestió d'Ajuts Universitaris i de Recerca (2009 SGR 695), and the Swiss Competence Centre for Materials Science and Technology (CCMX).

\section{References}

[1] J. Stöhr, H. C. Siegmann, Magnetism, from Fundamentals to Nanoscale Dynamics, Springer, Berlin, 2006.

[2] R. E. Camley, R. L. Stamps, Magnetic multilayers: spin configurations, excitations and giant magnetoresistance, J. Phys.: Condens. Matter 5 (23) (1993) 3727.

[3] B. Heinrich, J. F. Cochran, Adv. in Phys. 42 (1993) 523.

[4] E. Y. Tsymbal, I. Zutic, Handbook of spin transport and magnetism, CRC Press, Boca Raton, 2011.

[5] L. Bogani, W. Wernsdorfer, Molecular spintronics using single-molecule magnets, Nat. Mater. 7 (2008) 179-186.

[6] V. A. Dediu, L. E. Hueso, I. Bergenti, C. Taliani, Spin routes in organic semiconductors, Nat. Mater. 8 (2009) 707 - 716.

[7] M. N. Baibich, J. M. Broto, A. Fert, F. N. V. Dau, F. Petroff, P. Eitenne, G. Creuzet, A. Friederich, J. Chazelas, Phys. Rev. Lett. 61 (1988) 2472.

[8] G. Binasch, P. Grünberg, F. Saurenbach, W. Zinn, Phys. Rev. B 39 (1989) 4828.

[9] J. S. Moodera, L. R. Kinder, T. M. Wong, R. Meservey, Large magnetoresistance at room temperature in ferromagnetic thin film tunnel junctions, Phys. Rev. Lett. 74 (16) (1995) 3273.

[10] E. E. Fullerton, J. Jiang, S. Bader, Hard/soft magnetic heterostructures: model exchange-spring magnets, J. Magn. Magn. Mater. 200 (1) (1999) 392-404.

[11] J. Nogués, I. K. Schuller, Exchange bias, J. Magn. Magn. Mater. 192 (2) (1999) 203-232

[12] S. Schmaus, A. Bagrets, Y. Nahas, T. K. Yamada, A. Bork, M. Bowen, E. Beaurepaire, F. Evers, W. Wulfhekel, Giant magnetoresistance through a single molecule, Nat. Nanotechnol. 6 (3) (2011) 185-189.

[13] Z. Xiong, D. Wu, Z. V. Vardeny, J. Shi, Giant magnetoresistance in organic spin-valves, Nature 427 (6977) (2004) 821-824.

[14] K. V. Raman, A. M. Kamerbeek, A. Mukherjee, N. Atodiresei, T. K. Sen, P. Lazić, V. Caciuc, R. Michel, D. Stalke, S. K. Mandal, et al., Interface-engineered templates for molecular spin memory devices, $\mathrm{Na}$ ture 493 (7433) (2013) 509-513.

[15] A. Lodi Rizzini, C. Krull, T. Balashov, J. J. Kavich, A. Mugarza, P. S. Miedema, P. K. Thakur, V. Sessi, S. Klyatskaya, M. Ruben, S. Stepanow, P. Gambardella, Coupling single molecule magnets to ferromagnetic substrates, Phys. Rev. Lett. 107 (17) (2011) 177205-.

[16] A. Lodi Rizzini, C. Krull, T. Balashov, A. Mugarza, C. Nistor, F. Yakhou, V. Sessi, S. Klyatskaya, M. Ruben, S. Stepanow, et al., Exchange biasing single molecule magnets: Coupling of tbpc 2 to antiferromagnetic layers, Nano Lett. 12 (11) (2012) 5703-5707.

[17] T. Suzuki, M. Kurahashi, X. Ju, Y. Yamauchi, Spin polarization of metal (mn, fe, cu, and $\mathrm{mg}$ ) and metal-free phthalocyanines on an fe(100) substrate, J. Phys. Chem. B 106 (44) (2002) 11553-11556.

[18] S. Javaid, M. Bowen, S. Boukari, L. Joly, J.-B. Beaufrand, X. Chen, Y. J. Dappe, F. Scheurer, J.-P. Kappler, J. Arabski, W. Wulfhekel, M. Alouani, E. Beaurepaire, Impact on interface spin polarization of molecular bonding to metallic surfaces, Phys. Rev. Lett. 105 (7) (2010) 077201-.

[19] C. Wäckerlin, J. Nowakowski, S.-X. Liu, M. Jaggi, D. Siewert, J. Girovsky, A. Shchyrba, T. Hählen, A. Kleibert, P. M. Oppeneer, et al., Two-dimensional supramolecular electron spin arrays, Advanced Materials 25 (17) (2013) 2404-2408.

[20] S. Lach, A. Altenhof, K. Tarafder, F. Schmitt, M. Ali, M. Vogel, J. Sauther, P. M. Oppeneer, C. Ziegler, et al., Metal-organic hybrid interface states of a ferromagnet/organic semiconductor hybrid junction as basis for engineering spin injection in organic spintronics, Adv. Funct. Mater. 22 (5) (2012) 989-997.

[21] E. Annese, J. Fujii, I. Vobornik, G. Panaccione, G. Rossi, Control of the magnetism of cobalt phthalocyanine by a ferromagnetic substrate, Phys. Rev. B 84 (2011) 174443.

[22] D. Klar, B. Brena, H. Herper, S. Bhandary, C. Weis, B. Krumme, C. Schmitz-Antoniak, B. Sanyal, O. Eriksson, H. Wende, Oxygen-tuned magnetic coupling of fe-phthalocyanine molecules to ferromagnetic co films, Phys. Rev. B 88 (22) (2013) 224424.

[23] C. Iacovita, M. V. Rastei, B. W. Heinrich, T. Brumme, J. Kortus, L. Limot, J. P. Bucher, Visualizing the spin of individual cobaltphthalocyanine molecules, Phys. Rev. Lett. 101 (11) (2008) 116602-.

[24] J. Brede, N. Atodiresei, S. Kuck, P. Lazić, V. Caciuc, Y. Morikawa, G. Hoffmann, S. Blügel, R. Wiesendanger, Spin- and energy-dependent 
tunneling through a single molecule with intramolecular spatial resolution, Phys. Rev. Lett. 105 (4) (2010) 047204.

[25] A. Scheybal, T. Ramsvik, R. Bertschinger, M. Putero, F. Nolting, T. Jung, Induced magnetic ordering in a molecular monolayer, Chem. Phys. Lett. 411 (1-3) (2005) $214-220$.

[26] D. Chylarecka, C. Wckerlin, T. K. Kim, K. Mller, F. Nolting, A. Kleibert, N. Ballav, T. A. Jung, Self-assembly and superexchange coupling of magnetic molecules on oxygen-reconstructed ferromagnetic thin film, Journal of Physical Chemistry Letters 1 (9) (2010) 1408-1413.

[27] C. Wäckerlin, D. Chylarecka, A. Kleibert, K. Müller, C. Iacovita, F. Nolting, T. A. Jung, N. Ballav, Controlling spins in adsorbed molecules by a chemical switch, Nat. Commun. 1 (5) (2010) 61-.

[28] C. Wäckerlin, K. Tarafder, D. Siewert, J. Girovsky, T. Hählen, C. Iacovita, A. Kleibert, F. Nolting, T. A. Jung, P. M. Oppeneer, et al., Onsurface coordination chemistry of planar molecular spin systems: novel magnetochemical effects induced by axial ligands, Chemical Science 3 (11) (2012) 3154-3160.

[29] H. Wende, M. Bernien, J. Luo, C. Sorg, N. Ponpandian, J. Kurde, J. Miguel, M. Piantek, X. Xu, P. Eckhold, W. Kuch, K. Baberschke, P. M. Panchmatia, B. Sanyal, P. M. Oppeneer, O. Eriksson, Substrateinduced magnetic ordering and switching of iron porphyrin molecules, Nat. Mater. 6 (7) (2007) 516-520.

[30] M. Bernien, J. Miguel, C. Weis, M. E. Ali, J. Kurde, B. Krumme, P. M. Panchmatia, B. Sanyal, M. Piantek, P. Srivastava, K. Baberschke, P. M. Oppeneer, O. Eriksson, W. Kuch, H. Wende, Tailoring the nature of magnetic coupling of fe-porphyrin molecules to ferromagnetic substrates, Phys. Rev. Lett. 102 (4) (2009) 047202-

[31] C. F. Hermanns, K. Tarafder, M. Bernien, A. Krüger, Y.-M. Chang, P. M Oppeneer, W. Kuch, Magnetic coupling of porphyrin molecules through graphene, Advanced Materials 25 (25) (2013) 3473-3477.

[32] J. Klanke, E. Rentschler, K. Medjanik, D. Kutnyakhov, G. Schönhense, S. Krasnikov, I. V. Shvets, S. Schuppler, P. Nagel, M. Merz, H. J. Elmers, Beyond the heisenberg model: Anisotropic exchange interaction between a cu-tetraazaporphyrin monolayer and $\mathrm{Fe}_{3} \mathrm{O}_{4}(100)$, Phys. Rev. Lett. 110 (2013) 137202.

[33] J. Schwöbel, Y. Fu, J. Brede, A. Dilullo, G. Hoffmann, S. Klyatskaya, M. Ruben, R. Wiesendanger, Real-space observation of spinsplit molecular orbitals of adsorbed single-molecule magnets, Nature communications 3 (2012) 953

[34] D. Klar, S. Klyatskaya, A. Candini, B. Krumme, K. Kummer, P. Ohresser, V. Corradini, V. de Renzi, R. Biagi, L. Joly, et al., Antiferromagnetic coupling of tbpc2 molecules to ultrathin ni and co films, Beilstein journal of nanotechnology 4 (1) (2013) 320-324.

[35] L. Malavolti, L. Poggini, L. Margheriti, D. Chiappe, P. Graziosi, B. Cortigiani, V. Lanzilotto, F. B. de Mongeot, P. Ohresser, E. Otero, F. Choueikani, P. Sainctavit, B. I., D. V. A., M. Mannini, R. Sessoli, Magnetism of tbpc 2 smms on ferromagnetic electrodes used in organic spintronics, Chem. Commun. 49 (98) (2013) 11506-11508

[36] A. Mugarza, R. Robles, C. Krull, R. Korytar, N. Lorente, P. Gambardella, Electronic and magnetic properties of molecule-metal interfaces: Transition-metal phthalocyanines adsorbed on ag (100), Phys. Rev. B 85 (15) (2012) 155437

[37] P. H. Lippel, R. J. Wilson, M. D. Miller, C. Wöll, S. Chiang, Highresolution imaging of copper-phthalocyanine by scanning-tunneling microscopy, Phys. Rev. Lett. 62 (1989) 171-174.

[38] A. Mugarza, N. Lorente, P. Ordejón, C. Krull, S. Stepanow, M.-L. Bocquet, J. Fraxedas, G. Ceballos, P. Gambardella, Orbital specific chirality and homochiral self-assembly of achiral molecules induced by charge transfer and spontaneous symmetry breaking, Phys. Rev. Lett. 105 (11) (2010) 115702.

[39] W. Auwärter et al., Self-assembly and conformation of tetrapyridylporphyrin molecules on ag(111), J. Chem. Phys. 124 (19) (2006) 194708

[40] D. Ecija, M. Trelka, C. Urban, P. d. Mendoza, E. Mateo-Marti, C. Rogero, J. A. Martin-Gago, A. M. Echavarren, R. Otero, J. M. Gallego, R. Miranda, Molecular conformation, organizational chirality, and iron metalation of meso-tetramesitylporphyrins on copper(100), J. Phys Chem. C 112 (24) (2008) 8988-8994.

[41] A. Mugarza, C. Krull, R. Robles, S. Stepanow, G. Ceballos, P. Gambardella, Spin coupling and relaxation inside molecular junctions, Nature Communications 2:490 (2011) 1
[42] S. Stepanow, P. Miedema, A. Mugarza, G. Ceballos, P. Moras, J. C. Cezar, C. Carbone, F. M. F. de Groot, P. Gambardella, Charge-transfer correlation effects on the spin state of magnetic molecules on metals, Phys. Rev. B 83 (2011) 220401.

[43] S. Bhandary, B. Brena, P. M. Panchmatia, I. Brumboiu, M. Bernien, C. Weis, B. Krumme, C. Etz, W. Kuch, H. Wende, O. Eriksson, B. Sanyal, Manipulation of spin state of iron porphyrin by chemisorption on magnetic substrates, Phys. Rev. B 88 (2013) 024401.

[44] N. Ishikawa, M. Sugita, T. Ishikawa, S.-y. Koshihara, Y. Kaizu, Lanthanide double-decker complexes functioning as magnets at the singlemolecular level, J. Am. Chem. Soc. 125 (29) (2003) 8694-8695.

[45] F. Branzoli, P. Carretta, M. Filibian, G. Zoppellaro, M. J. Graf, J. R. Galan-Mascaros, O. Fuhr, S. Brink, M. Ruben, Spin dynamics in the negatively charged terbium (iii) bis-phthalocyaninato complex, J. Am. Chem. Soc. 131 (12) (2009) 4387-4396.

[46] S. Stepanow, J. Honolka, P. Gambardella, L. Vitali, N. Abdurakhmanova, T.-C. Tseng, S. Rauschenbach, S. L. Tait, V. Sessi, S. Klyatskaya, M. Ruben, K. Kern, Spin and orbital magnetic moment anisotropies of monodispersed bis(phthalocyaninato)terbium on a copper surface, J. Am. Chem. Soc. 132 (34) (2010) 11900-11901.

[47] L. Margheriti, D. Chiappe, M. Mannini, P. Car, P. Sainctavit, M.-A. Arrio, F. B. de Mongeot, J. C. Cezar, F. M. Piras, A. Magnani, E. Otero, A. Caneschi, R. Sessoli, X-ray detected magnetic hysteresis of thermally evaporated terbium double-decker oriented films, Adv. Mater. 22 (48) (2010) 5488-5493

[48] J. Jiang, D. K. Ng, A decade journey in the chemistry of sandwich-type tetrapyrrolato- rare earth complexes, Acc. Chem. Res. 42 (1) (2008) 79 88.

[49] H. Wang, K. Wang, Y. Bian, J. Jiang, N. Kobayashi, Mixed (phthalocyaninato)(porphyrinato) heterometal complexes with sandwich quadruple-decker molecular structure, Chem. Comm. 47 (24) (2011) 6879-6881.

[50] P. Gambardella, S. Dhesi, S. Gardonio, C. Grazioli, P. Ohresser, C. Carbone, Phys. Rev. Lett. 88 (2002) 047202.

[51] H. Brune, P. Gambardella, Magnetism of individual atoms adsorbed on surfaces, Surf. Sci. 603 (10) (2009) 1812-1830.

[52] F. Branzoli, P. Carretta, M. Filibian, M. Graf, S. Klyatskaya, M. Ruben, F. Coneri, P. Dhakal, Spin and charge dynamics in $\left[\mathrm{TbPc}_{2}\right]^{0}$ and $\left[\mathrm{DyPc}_{2}\right]^{0}$ single-molecule magnets, Phys. Rev. B 82 (13) (2010) 134401.

[53] K. Kasuga, M. Ando, H. Morimoto, M. Isa, Preparation of new phthalocyanine complexes of yttrium(iii) and some lanthanoid(iii) ions, Chem. Lett.

[54] F. Huang, M. T. Kief, G. J. Mankey, R. F. Willis, Magnetism in the fewmonolayers limit: A surface magneto-optic kerr-effect study of the magnetic behavior of ultrathin films of co, ni, and co-ni alloys on cu(100) and cu(111), Phys. Rev. B 49 (6) (1994) 3962-

[55] B. Schulz, K. Baberschke, Crossover from in-plane to perpendicular magnetization in ultrathin ni/cu(001) films, Phys. Rev. B 50 (18) (1994) 13467-.

[56] I. Sebastian, T. Bertrams, K. Meinel, H. Neddermeyer, Scanning tunnelling microscopy on the growth and structure of nio(100) and coo(100) thin films, Faraday Discuss. 114 (1999) 129-140.

[57] E. Goering, A. Fuss, W. Weber, J. Will, G. Schütz, Element specific xray magnetic circular dichroism magnetization curves using total electron yield, J. Appl. Phys. 88 (10) (2000) 5920-5923.

[58] P. Gambardella, A. Dallmeyer, K. Maiti, M. Malagoli, W. Eberhardt, K. Kern, C. Carbone, Ferromagnetism in one-dimensional monatomic metal chains, Nature 416 (2002) 301.

[59] P. Gambardella, J. Phys.: Condens. Matter 15 (2003) S2533.

[60] J. Dresselhaus, D. Spanke, F. U. Hillebrecht, E. Kisker, G. van der Laan, J. B. Goedkoop, N. B. Brookes, Antiferromagnetic coupling of mn adsorbates to fe(100), Phys. Rev. B 56 (9) (1997) 5461-5467.

[61] G. Dufour, C. Poncey, F. Rochet, H. Roulet, S. Iacobucci, M. Sacchi, F. Yubero, N. Motta, M. N. Piancastelli, A. Sgarlata, M. D. Crescenzi, Metal phthalocyanines $(\mathrm{mpc}, \mathrm{m}=\mathrm{ni}, \mathrm{cu})$ on $\mathrm{cu}(001)$ and $\mathrm{si}(001)$ surfaces studied by xps, xas and stm, J. El. Spec. Rel. Phen. 76 (1995) $219-224$.

[62] L. Vitali, S. Fabris, A. M. Conte, S. Brink, M. Ruben, S. Baroni, K. Kern, Electronic structure of surface-supported bis(phthalocyaninato) terbium(iii) single molecular magnets, Nano Lett. 8 (10) (2008) 3364 3368.

[63] R. Nünthel, T. Gleitsmann, P. Poulopoulos, A. Scherz, J. Lindner, E. Ko- 
subek, C. Litwinski, Z. Li, H. Wende, K. Baberschke, S. Stolbov, T. S. Rahman, Epitaxial growth of ni on cu(l 001$)$ with the assistance of osurfactant and its magnetism compared to ni/cu(0 0 1), Surf. Sci. 531 (2003) $53-67$.

[64] P. L. Cook, X. Liu, W. Yang, F. J. Himpsel1, X-ray absorption spectroscopy of biomimetic dye molecules for solar cells, J. Chem. Phys 131 (2009) 194701.

[65] J. B. Goedkoop, B. T. Thole, G. van der Laan, G. A. Sawatzky, F. M. F. de Groot, J. C. Fuggle, Calculations of magnetic x-ray dichroism in the $3 \mathrm{~d}$ absorption spectra of rare-earth compounds, Phys. Rev. B 37 (4) (1988) 2086-2093.

[66] K. Katoh, T. Komeda, M. Yamashita, Surface morphologies, electronic structures, and kondo effect of lanthanide (iii)-phthalocyanine molecules on au (111) by using stm, sts and fet properties for next generation devices, Dalton Trans. 39 (20) (2010) 4708-4723.

[67] C. G. Barraclough, R. L. Martin, S. Mitra, R. C. Sherwood, Paramagnetic anisotropy, electronic structure, and ferromagnetism in spin $\mathrm{s}=32$ manganese(ii) phthalocyanine, The Journal of Chemical Physics 53 (5) (1970) 1638-1642

[68] J. Wang, Y. Shi, J. Cao, R. Wu, Magnetization and magnetic anisotropy of metallophthalocyanine molecules from the first principles calculations, Appl. Phys. Lett. 94 (12) (2009) 122502.

[69] F. Djeghloul, F. Ibrahim, M. Cantoni, M. Bowen, L. Joly, S. Boukari, P. Ohresser, F. Bertran, P. Le Fèvre, P. Thakur, et al., Direct observation of a highly spin-polarized organic spinterface at room temperature, Sci. Rep. 3.

[70] S. Stepanow, A. L. Rizzini, C. Krull, J. Kavich, J. C. Cezar, F. YakhouHarris, P. M. Sheverdyaeva, P. Moras, C. Carbone, G. Ceballos, A. Mugarza, P. Gambardella, Spin tuning of electron-doped metalphthalocyanine layers, J. Am. Chem. Soc. 136 (2014) 5451-5459.

[71] A. Postnikov, V. Anisimov, V. Gubanov, Cluster approach to magnetic impurities in metals: Application to cumn, timn, nimn, nbfe and mofe, J. Mag. Magn. Mater. 39 (3) (1983) 295 - 308.

[72] X. Sun, B. Wang, Y. Yamauchi, Electronic structure and spin polarization of metal (mn, fe, cu) phthalocyanines on an fe(100) surface by firstprinciples calculations, J. Phys. Chem. C 116 (35) (2012) 18752-18758.

[73] N. Ishikawa, Single molecule magnet with single lanthanide ion, Polyhedron 26 (9-11) (2007) 2147 - 2153, proceedings of the 10th International Conference on Molecule-based Magnets (ICMM 2006) - Victoria, B.C., Canada, August 13-17, 2006, ICMM 2006.

[74] N. Ishikawa, M. Sugita, T. Okubo, N. Tanaka, T. Iino, Y. Kaizu, Determination of ligand-field parameters and f-electronic structures of doubledecker bis(phthalocyaninato)lanthanide complexes, Inorg. Chem. 42 (7) (2003) 2440-2446.

[75] N. Ishikawa, M. Sugita, T. Ishikawa, S.-y. Koshihara, Y. Kaizu, Mononuclear lanthanide complexes with a long magnetization relaxation time at high temperatures: A new category of magnets at the single-molecular level, J. Phys. Chem. B 108 (31) (2004) 11265-11271.

[76] M. Gonidec, R. Biagi, V. Corradini, F. Moro, V. De Renzi, U. del Pennino, D. Summa, L. Muccioli, C. Zannoni, D. B. Amabilino, J. Veciana, Surface supramolecular organization of a terbium(iii) doubledecker complex on graphite and its single molecule magnet behavior, J. Am. Chem. Soc. 133 (17) (2011) 6603-6612.

[77] N. Ishikawa, M. Sugita, N. Tanaka, T. Ishikawa, S. Koshihara, Y. Kaizu, Upward temperature shift of the intrinsic phase lag of the magnetization of bis(phthalocyaninato)terbium by ligand oxidation creating an $\mathrm{s}=1 / 2$ spin, Inorg. Chem. 43 (18) (2004) 5498-5500.

[78] N. Ishikawa, M. Sugita, W. Wernsdorfer, Quantum tunneling of magnetization in lanthanide single-molecule magnets: Bis(phthalocyaninato)terbium and bis(phthalocyaninato)dysprosium anions, Angew. Chem. Int. Ed. 44 (19) (2005) 2931-2935.

[79] J. M. Baker, Interactions between ions with orbital angular momentum in insulators, Rep. Prog. Phys. 34 (1) (1971) 109.

[80] P. H. Christensen, S. Mrup, On the magnetic dipole fields at surface atoms, J. Mag. Magn. Mater. 35 (1-3) (1983) 130 - 132

[81] C. Krull, R. Robles, A. Mugarza, P. Gambardella, Site-and orbitaldependent charge donation and spin manipulation in electron-doped metal phthalocyanines, Nat. Mater. 12 (4) (2013) 337-343.

[82] R. Robles, N. Lorente, H. Isshiki, J. Liu, K. Katoh, B. K. Breedlove, M. Yamashita, T. Komeda, Spin doping of individual molecules by using single-atom manipulation, Nano Lett. 12 (7) (2012) 3609-3612.
[83] T. Komeda, H. Isshiki, J. Liu, Y.-F. Zhang, N. Lorente, K. Katoh, B. K. Breedlove, M. Yamashita, Observation and electric current control of a local spin in a single-molecule magnet, Nat. Comm. 2 (2011) 217.

[84] P. Petit, P. Turek, J.-J. André, R. Even, J. Simon, R. Madru, M. Al Sadoun, G. Guillaud, M. Maitrot, Molecular semiconductors: Phthalocyanine radicals. magnetic and electrical properties, field effect transistors, Synth. Met. 29 (2) (1989) 59-64.

[85] D. Gryko, J. Li, J. R. Diers, K. M. Roth, D. F. Bocian, W. G. Kuhr, J. S. Lindsey, Studies related to the design and synthesis of a molecular octal counterelectronic supplementary information (esi) available: Absorption, ld-ms and $1 \mathrm{~h} \mathrm{nmr}$ spectra for each triple decker; $1 \mathrm{~h} \mathrm{nmr} \mathrm{spectra} \mathrm{for} \mathrm{precursors} \mathrm{to} \mathrm{triple} \mathrm{deckers.} \mathrm{see} \mathrm{http://www.} \mathrm{rsc.}$ org/suppdata/jm/b0/b008224o, J. Mater. Chem. 11 (4) (2001) 1162 1180.

[86] N. Ishikawa, S. Otsuka, Y. Kaizu, The effect of the ff interaction on the dynamic magnetism of a coupled $4 \mathrm{f} 8$ system in a dinuclear terbium complex with phthalocyanines, Angew. Chem. Intl. Ed. 44 (5) (2005) 731-733.

[87] K. Katoh, T. Kajiwara, M. Nakano, Y. Nakazawa, W. Wernsdorfer, N. Ishikawa, B. K. Breedlove, M. Yamashita, Magnetic relaxation of single-molecule magnets in an external magnetic field: An ising dimer of a terbium (iii)-phthalocyaninate triple-decker complex, Chem. Eur. J. 17 (1) (2011) 117-122.

[88] G. Guillaud, M. Al Sadoun, M. Maitrot, J. Simon, M. Bouvet, Fieldeffect transistors based on intrinsic molecular semiconductors, Chemical Physics Letters 167 (6) (1990) 503-506.

[89] Y. Chen, W. Su, M. Bai, J. Jiang, X. Li, Y. Liu, L. Wang, S. Wang, High performance organic field-effect transistors based on amphiphilic tris(phthalocyaninato) rare earth triple-decker complexes, J. Am. Chem. Soc. 127 (45) (2005) 15700-15701.

[90] Z. Liu, A. A. Yasseri, J. S. Lindsey, D. F. Bocian, Molecular memories that survive silicon device processing and real-world operation, Science 302 (5650) (2003) 1543-1545.

[91] N. Ishikawa, T. Iino, Y. Kaizu, Interaction between f-electronic systems in dinuclear lanthanide complexes with phthalocyanines, Journal of the American Chemical Society 124 (38) (2002) 11440-11447.

[92] K. Takano, R. H. Kodama, A. E. Berkowitz, W. Cao, G. Thomas, Interfacial uncompensated antiferromagnetic spins: Role in unidirectional anisotropy in polycrystalline $\mathrm{Ni}_{81} \mathrm{Fe}_{19} / \mathrm{CoO}$ bilayers, Phys. Rev. Lett. 79 (6) (1997) 1130-1133.

[93] H. Ohldag, A. Scholl, F. Nolting, E. Arenholz, S. Maat, A. T. Young, M. Carey, J. Stöhr, Correlation between exchange bias and pinned interfacial spins, Phys. Rev. Lett. 91 (1) (2003) 017203-.

[94] J. Noguès, S. Stepanow, A. Bollero, J. Sort, B. Dieny, F. Nolting, P. Gambardella, Simultaneous in-plane and out-of-plane exchange bias using a single antiferromagnetic layer resolved by x-ray magnetic circular dichroism, Appl. Phys. Lett. 95 (15) (2009) 152515-3.

[95] P. Gambardella, S. Stepanow, A. Dmitriev, J. Honolka, F. M. F. de Groot, M. Lingenfelder, S. S. Gupta, D. D. Sarma, P. Bencok, S. Stanescu, S. Clair, S. Pons, N. Lin, A. P. Seitsonen, H. Brune, J. V. Barth, K. Kern, Supramolecular control of the magnetic anisotropy in two-dimensional high-spin fe arrays at a metal interface, Nat. Mater. 8 (3) (2009) 189 193.

[96] T. Ambrose, C. L. Chien, Finite-size effects and uncompensated magnetization in thin antiferromagnetic coo layers, Phys. Rev. Lett. 76 (10) (1996) 1743-1746.

[97] S. I. Csiszar, M. W. Haverkort, Z. Hu, A. Tanaka, H. H. Hsieh, H.-J. Lin, C. T. Chen, T. Hibma, L. H. Tjeng, Controlling orbital moment and spin orientation in coo layers by strain, Phys. Rev. Lett. 95 (18) (2005) $187205-$

[98] B. T. Jonker, J. J. Krebs, G. A. Prinz, Growth and magnetic characterization of mn films and superlattices on ag(001), Phys. Rev. B 39 (1989) 1399-1402.

[99] S. Blügel, P. H. Dederichs, Ferromagnetism and Antiferromagnetism of 3 d Metal Overlayers on Noble-Metal Substrates, Europhys. Lett. 9 (6) (1989) 597-602.

100] P. Schieffer, M.-H. Tuilier, M.-C. Hanf, C. Krembel, G. Gewinner, Sexafs investigation of bct $\mathrm{mn}$ grown epitaxially on ag(001) at room temperature, Surf. Sci. 422 (13) (1999) 132-140.

[101] T. Massalski, J. Murray, L. Bennett, H. Baker, Binary alloy phase diagrams, no. v. 2 in Binary Alloy Phase Diagrams, 1986. 
[102] P. Schieffer, M. Hanf, C. Krembel, G. Gewinner, Formation of c(22) mnag superficial bilayer alloys on $\mathrm{ag}(001)$ : role of thermally activated surface atomic exchange and ordering, Surf. Sci. 446 (3) (2000) 175 186.

[103] P. Schieffer, C. Krembel, M.-C. Hanf, M.-H. Tuilier, P. Wetzel, G. Gewinner, K. Hricovini, High spin state of $\mathrm{mn}$ in an ideal monolayer on ag(001), Eur. Phys. J. B 8 (2) (1999) 165-168.

[104] P. Krüger, O. Elmouhssine, C. Demangeat, J. C. Parlebas, Magnetic structures of bct manganese in the bulk and at the (001) surface, Phys. Rev. B 54 (9) (1996) 6393-6400.

[105] J. Hafner, D. Spišák, Ab initio investigation of the magnetism of tetragonal mn: Bulk, surface, ultrathin films, and multilayers, Phys. Rev. B 72 (2005) 144420.

[106] W. Jauch, M. Reehuis, H. J. Bleif, F. Kubanek, P. Pattison, Crystallographic symmetry and magnetic structure of coo, Phys. Rev. B 64 (5) (2001) 052102-

[107] P. Gambardella, H. Brune, S. S. Dhesi, P. Bencok, S. R. Krishnakumar, S. Gardonio, M. Veronese, C. Grazioli, C. Carbone, Paramagnetic mn impurities on ge and gaas surfaces, Phys. Rev. B 72 (4) (2005) 045337-.

[108] O. Rader, W. Gudat, D. Schmitz, C. Carbone, W. Eberhardt, Magnetic circular x-ray dichroism of submonolayer mn on fe(100), Phys. Rev. B 56 (1997) 5053-5056.

[109] W. L. O'Brien, B. P. Tonner, Magnetic properties of $\mathrm{Mn} / \mathrm{Cu}(001)$ and $\mathrm{Mn} / \mathrm{Ni}(001)$ surface alloys, Phys. Rev. B 51 (1995) 617-620.

[110] J. W. Cai, K. Liu, C. L. Chien, Exchange coupling in the paramagnetic state, Phys. Rev. B 60 (1) (1999) 72-75. 